Mittheilungen aus dem chemischen Institut der

Universität Greifswald.

I. Ueber Beziehungen

zwischen Constitution und Beständigkeit bei den Condensationsproducten organischer Basen mit substituirten Oxybenzylbromiden;

ron $K$. Auwers.

(Eingelanfen am 11. November 1905.)

Bereits zu Begimu ${ }^{1}$ ) meiner Studien über die abnorm reactionsfähigen Oxybenzylbromide und verwandte Verbindungen, für die ich später ${ }^{2}$ ) den Namen Pseudophenole vorgeschlagen habe, beobachtete ich, dass verschiedene Basen, die durch Umsetzung derartiger Körper mit Aminen entstehen, durch eine unerwartete Zersetzlichkeit ausgezeichnet sind, während andere recht beständige Körper sind. So spaltete beispielsweise das Piperidinderivat

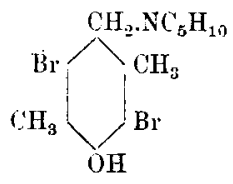

auf dem Wasserbade rasch Piperidin ab und ging in eine stickstofffreie Substanz über, und noch leichter zersetzte sich die aus Pyridin und Pseudocumenoltribromid gewonnene Verbindung

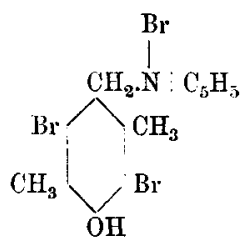

1) Ber. d. deutsch. chem. Ges. 28, 2888 (1895).

2) Ber. d. deutsch, chem. Ges. 34, 4256 (1901). 
unter dem Einflusse der verschiedensten Agentien. Dagegen wurden an dem entsprechenden Anilidoderivate keine ungewöbnlichen Eigenschaften beobachtet.

Eine Ausdebnung dieser Versuche auf die Abkömmlinge anderer Basen liess weitere merkwürdige Verschiedenheiten erkennen, und je weiter die Untersuchungen über die Pseudophenole fortschritten, um so grösser wurde die Zahl vereinzelter Beobachtungen, die sich auf dieses Gebiet bezogen. Dabei stellten sich im Laufe der Zeit so auffallende Gegensätze in dem Verhalten der einzelnen Condensationsproducte, die doch alle nach dem gleichen Schema gebaut waren, heraus, dass eine systematische Untersuchung dieser Verhältnisse am Platze zu sein schien.

Schon die gelegentlich hier und da gemachten Beobachtungen hatten gelehrt, dass der Grad von Beständigkeit bei diesen Condensationsproducten sowohl von der Natur der an ihrem Aufbau betheiligten Base, wie von der Constitution des betreffenden Pseudophenols oder Phenols abhängt. Man musste daher einmal die Reactionsproducte einer grösseren Zahl von Basen mit einigen Bromiden darstellen, und zweitens möglichst viel verschiedene Vertreter dieser Oxybenzylbromide mit einigen ausgewählten Basen condensiren, um das nöthige Material für die Untersuchung zu sammeln. Diese Verbindungen selbst waren dann unter gleichen Bedingungen der Einwirkung bestimmter Agentien zu unterwerfen, damit man auf diese Weise ihre relative Beständigkeit feststellen und wenn möglich den Zusammenhang zwischen ihr und der Constitution der Körper ermitteln konnte.

Gemeinsam mit mehreren Mitarbeitern habe ich mich im Laufe der letzten Jahre bemüht, dieses Problem zu lösen; im Folgenden gebe ich einen Ueberblick über die bisher dabei erzielten Ergebnisse.

Ein erheblicher Theil der Versuche ist von den Herren C. Kipke und A. Schrenk angestellt worden, denen ich für ihre fleissige und geschickte Beihülfe meinen besten Dank sage. 
Auch meinem Assistenten, Herrn Dr. C. Roever, der bei dem vorläufigen Abschluss dieser Untersuchungen durch Darstellung vieler Präparate und Ausführung zahlreicher analytischer Bestimmungen eifrig mitgewirkt hat, bin ich sehr verbunden. Vor allen aber gebührt mein wärmster Dank meinem bisherigen Assistenten, Herrn Dr. 0. Schröter, der nicht nur eine grosse Schaar der erforderlichen neuen Verbindungen dargestellt und zuerst untersucht, sondern auch die ein ungewöhnliches Maass von Geduld erfordernde Aufgabe übernommen hat, den grössten Theil der von anderer Seite angestellten Versuche, oft mehrfach, $z u$ wiederholen und die einzelnen Beobachtungen nachzuprüfen. Seiner Gewissenhaftigkeit und unermüdlichen Ausdauer habe ich es in erster Linie zu danken, dass diese weitschichtige und mühevolle Untersuchung $\mathrm{zu}$ einigen bestimmten und sicheren Ergebnissen geführt hat, wenn auch ein völlig befriedigender Abschluss noch nicht erzielt werden konnte.

\section{Beschaffung des Materials.}

Folgende 17 Phenole und Pseudophenole sind für die Untersuchung herangezogen worden:

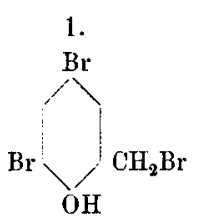

5.<smiles>BrCC(Br)C(Br)Br</smiles>

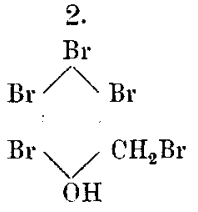

6.<smiles>CC(O)C(Br)Br</smiles>

3.<smiles>CCC(O)Br</smiles>

7.<smiles>CC(Br)Br</smiles>

4.<smiles>CC(Br)Br</smiles>

8.<smiles>Br[Hg]Br</smiles><smiles>CC(O)[C@H](Br)CCC(Br)[Hg]Br</smiles> 

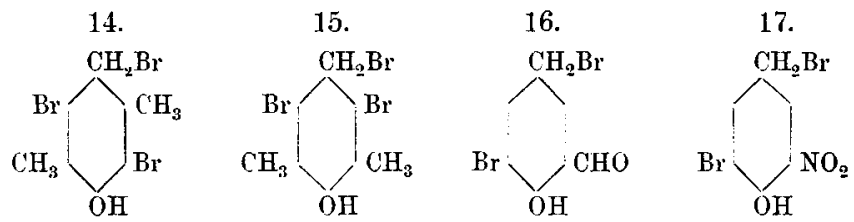

Der grösste Theil dieser Verbindungen war bereits bekannt; nur wenige sind für die Zwecke dieser Arbeit zum ersten Male dargestellt worden. Aber ron den bekannten Bromiden war eine ganze Anzahl früher nur in kleinen Quantitäten nach umständlichen und kostspieligen Verfahren gewonnen worden, und es galt nun, ergiebigere und wohlfeilere Darstellungsweisen aufzufinden. In manchen Fällen ist dies gelungen; in anderen sind dagegen alle Bemühungen erfolglos geblieben. Einige dieser schwer zugänglichen Substanzen konnten daher nicht in dem gewünschten Umfange untersucht werden.

An Basen kamen zur Verwendung: Ammoniak, Methyl-, Aethyl- und Benzylamin, Diäthyl- und Diamylamin, Anilin und Methylanilin, $\beta$-Naphtylamin, Piperidin, Pyridin und Chinolin.

Nur die beiden Tribromderivate des Pseudocumenols und Mesitols - Nr. 14 und 15 der obigen Zusammenstellung sind mit allen diesen Basen condensirt worden. An diesen beiden Reihen von Verbindungen sollte in erster Linie der Einfluss, den die Natur der basischen Componente auf die Beständigkeit der Producte ausübt, ermittelt werden. Gewählt wurden diese beiden Bromide, weil bei ihren Derivaten die Unterschiede im Verhalten gegen die verschiedenen Agentien besonders deutlich hervortreten, und beide leicht in grösseren Mengen $z u$ beschafien sind.

Die meisten anderen Bromide sind nur mit Metbyl- und Benzylamin, Diamylamin und Piperidin combinirt worden, soweit genügende Mengen beschafft werden konnten; in einigen wenigen Fällen hat man sich auf die Untersuchung eines einzigen oder zweier Condensationsproducte eines Bromides beschränken müssen. 
Ausserdem wurden zu Vergleichszwecken einige Condensationsproducte von Basen mit Methyläthern und Acetylverbindungen solcher Bromide dargestellt.

Das allgerneine Verfahren zur Gewinnung aller dieser Derivate war im Prinzip äusserst einfach, denn man brauchte in der Regel nur passende Mengen von Bromid und Amin in einem geeigneten Lösungsmittel auf einander einwirken zu lassen, - bei Pseudophenolen in der Kälte, bei Phenolen meist in der Hitze - und dann die entstandene Base von dem als Nebenproduct gebildeten bromwasserstoffsauren Amin zu trennen. Die praktische Durchführung der Reaction bot jedoch häufig recht erhebliche Schwierigkeiten.

Günstige Ergebnisse wurden gewöhnlich erzielt, wenn das Condensationsproduct sich in Folge geringer Löslichkeit zusammen mit dem Bromhydrat der angewandten Base aus dem Lösungsmittel - meist Benzol oder Aether - ausschied. Man konnte dann das beigemengte Salz durch warmes Wasser oder andauerndes Schütteln mit kaltem Wasser ausziehen und darauf den Rückstand durch Umkrystallisiren oder Auflösen in verdünnter Lauge und Wiederfällen durch Kohlensäure reinigen.

Blieb das Reactionsproduct in Lösung, so filtrirte man von dem ausgeschiedenen Salz ab und liess das Filtrat, wenn die neue Base leidlich beständig war, freiwillig eindunsten. Die weitere Behandlung des Rückstandes gestaltete sich senr verschieden. In vereinzelten Fällen konnte er direct umkrystallisirt werden. Meist mussten zunächst durch Anrühren mit Wasser oder sehr verdünnter Essigsäure kleine Mengen unveränderten Amins oder dessen Bromhydrats weggeschafft, oder durch Verreiben mit wenig Alkohol, Methylalkohol, Benzol oder Ligroïn harzige Verunreinigungen beseitigt werden. Liess sich auch dann das Reactionsproduct noch nicht umkrystallisiren, so verwandelte man es in sein Chlorhydrat und schied es aus liesem wieder ab, oder fällte es aus alkalischer Lösung durch Kohlensäure. War dagegen das Condensationsproduct so unbeständig, dass es sich bereits beim Eindunsten der Lösung zu 
zersetzen begann, so wurde es aus dem benzolischen oder ätherischen Filtrate durch trocknen Chlorwasserstoff gefällt und aus diesem durch Verreiben mit verdünnter Sodalösung unter Kühlung in Freiheit gesetzt. Auch für weniger zersetzliche Verbindungen hat sich dieser, Weg in manchen Fällen als rortheilhaft erwiesen.

Einzelne Basen haben sich trotz wiederholter Anwendung der verschiedenen Reinigungsoperationen nicht in krystallisirtem Zustande gewinnen lassen und mussten daher in Form ihrer Salze untersucht werden. Andere, die bei den Analysen dauernd unbefriedigende Werthe lieferten, wurden von der Untersuchung ausgeschlossen.

Nähere Angaben über die Darstellung und Eigenschaften der verschiedenen Verbindungen finden sich in den folgenden Mit theilungen.

Was die Zusammensetzung der Condensationsproducte betrifft, so wurde im Allgemeinen die bereits früher aufgestellte Regel bestätigt gefunden, dass aus aliphatischen primären Aminen Körper vom Typus Ar. $\mathrm{CH}_{2}-\mathrm{N}(\mathrm{Alph})-\mathrm{CH}_{2}-\mathrm{Ar}$ entstehen, aus aromatischen dagegen Substanzen von der Formel

$$
\text { Ar.CH.NH.Ar. }
$$

Eine eigenthümliche Regelmässigkeit wurde bei den $\mathrm{Ab}$ kömmlingen des Benzylamins beobachtet. Mit den drei Bromiden, die sich vom Pseudocumenol ableiten:
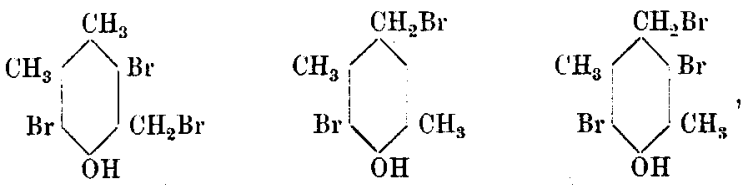

bildete es regelmässig die tertiären Basen,

$$
\begin{array}{r}
\mathrm{CH}_{2} \cdot \mathrm{C}_{6} \mathrm{H}_{5} \\
\mathrm{Ar} . \mathrm{CH}_{2} \cdot \mathrm{N} \cdot \mathrm{CH}_{2} \cdot \mathrm{Ar},
\end{array}
$$

während es mit den anderen, nach dieser Richtung untersuchten Pseudophenolen: 
<smiles>CCCC(O)CBr</smiles><smiles>BrC[C@H](Br)[In]Br</smiles><smiles>BrC(Br)C(Br)Br</smiles><smiles>C[C@H]1C[C@H](Br)[C@H](Br)[C@H]1Br</smiles><smiles></smiles><smiles>CCC(Br)C(C)Br</smiles>

stets zu secundären Basen der Form Ar. $\mathrm{CH}_{2} \cdot \mathrm{NH} \mathrm{CH}_{2} \cdot \mathrm{C}_{6} \mathrm{H}_{5}$ zusammentrat. Namentlich mit den ihrer Constitution nach eng zusammengehörenden Tribromderivaten des Pseudocumenols und Mesitols sind die Versuche unter möglichst gleichen Bedingungen mehrfach wiederholt worden, da der Verlauf der Condensation vielleicht von kleinen Zufälligkeiten beeinflusst werden konnte; aber stets wurde der gleiche Unterschied beobachtet.

Besonders auffallend ist, dass die Erscheinung bei dem Ortho- und bei den Paraderivaten des Pseudocumenols in der gleichen Weise auftritt, also von der Stellung der Methyle zu dem eintretenden basischen Rest unabhängig zı sein scheint.

Bedingt in diesem Falle dem Anschein nach die Constitution des Bromids einen besonderen Verlauf der Reaction, so kann in gleicher Weise auch der Bau der einwirkenden Base einen bestimmenden Einfluss ausüben; wenigstens nimmt in dieser Beziehung unter den bisher untersuchten Basen das vic. $\mathrm{m}$-Xylidin eine charakteristische Sonderstellung ein, wie in der letzten der folgenden Arbeiten näher auseinandergesetzt werden wird.

Nicht selten entstehen übrigens bei der Umsetzung von Benzylamin mit Pseudophenolen Gemische, die je nach den Versuchsbedingungen bald mehr secundäre, bald mehr tertiäre Base enthalten. Aus diesem Grunde ist die Reindarstellung verschiedener Benzylaminderivate bisher noch nicht geglitickt. 


\section{Allgemeine Methode der Untersuchung.}

Von früheren gelegentlichen Beobachtungen her war bekannt, dass die einzelnen Condensationsproducte zum Theil gegen alkalische und saure Agentien eine sehr ungleiche Widerstandsfähigkeit besitzen. Es war daher nöthig, jede Verbindung, soweit genügende Substanzmengen zu beschaffen waren, einer systematischen Untersuchung unter gleichbleibenden Versuchsbedingungen zu unterwerfen.

Nach verschiedenen Vorversuchen blieb man dabei stehen, das Verhalten der Basen gegen kalte und heisse verdünnte Natronlauge, gegen kalte und heisse 100 procentige Essigsäure, gegen kochendes Essigsäureanhydrid und endlich gegen längeres Erhitzen auf dem Wasserbade zu prüfen.

In einigen wenigen Fällen wurde auch festgestellt, wie kochendes Wasser, Methyl- und Aethylalkohol, Toluol und andere Lösungsmittel bei verschiedenen Temperaturen einwirken.

Um vergleichbare Resultate zu gewinnen, führte man sämmtliche Versucbe möglichst genau nach folgendem Schema durch:

\section{Verhalten gegen Natronlauge.}

Bei den ersten Versuchsreiben wurde zehnprocentige Natronlauge angewendet. Als man erkannt hatte, dass diese im Allgemeinen zu kräftig wirkt und die Unterschiede in der Beständigkeit der Basen verdünnterer Lauge gegenüber deutlicher hervortreten, wurden die späteren Versuche meist mit fünfprocentiger Natronlauge durchgeführt; auch die früheren, soweit es nöthig erschien, mit der schwächeren Lauge wiederholt. In vereinzelten Fällen wurde auch zum Vergleich einprocentige Natronlauge benutzt.

Bei den Versuchen in der Kälte befeuchtete man 0,5 bis $1,0 \mathrm{~g}$ Substanz - nur vereinzelte Versuche wurden aus besonderen Gründen mit grösseren Mengen durchgeführt - in einem kleinen Erlenmeyer-Kolben mit wenigen Tropfen Alkohol, um den Angriff des Alkalis zu erleichtern, fügte $5 \mathrm{ccm}$ fünfprocentige Natronlauge hinzu, schüttelte, bis alles gelöst 
war und liess dann verstopft 24 Stunden - in einer Reihe älterer Versuche 18 Stunden - bei Zimmertemperatur stehen. Löste sich nicht alles klar auf, so fügte man nach und nach so viel Alkohol hinzu, als zur völligen Lösung eben nöthig war.

Bei den Versuchen in der Hitze wurde eine in gleicher Weise hergestellte Lösung - Zusatz von Alkohol war hierbei nur in seltensten Fällen nöthig - eine Stunde auf dem Drahtnetze uber freier Flamme am Steigrohre oder Rückflusskühler gekocht und dann abgekühlt.

In beiden Fällen verdünnte man nach Beendigung des Versuches die Flüssigkeit mit dem drei- bis vierfachen Volumen Wasser und fällte das Reactionsproduct durch Kohlensäure oder stark verdünnte Essigsäure aus; der Ueberschuss von Essigsäure wurde durch Sodalösung abgestumpft.

Erwies sich der abfiltrirte, gut mit Wasser gewaschene und auf Thon getrocknete Niederschlag bei der qualitativen Prüfung frei von Stickstoff, so war die ursprüngliche Base völlig zersetzt worden. Es lagen dann regelmässig Spaltungsproducte vor, die anfänglich für Stilbenderivate angesehen, später aber als Abkömmlinge des Diphenylmethans erkannt worden sind ${ }^{3}$ ). Sie werden im Folgenden schlechtweg als ,Diphenylmethanderivate" bezeichnet werden. Sofort völlig rein waren diese Zersetzungsproducte nie, konnten aber in den allermeisten Fällen durch Umkrystallisiren aus siedendem Benzol leicht gereinigt werden. Wo es irgend erforderlich schien, wurden diese Körper, ebenso wie alle anderen bei den Spaltungsversuchen erhaltenen, reinen Producte, überdies durch directen Vergleich mit Präparaten, die auf anderem Wege hergestellt worden waren, identificirt. Namentlich wurde Werth daranf gelegt, nach Möglichkeit die Schmẹlzpunkte von Gemischen der zu vergleichenden Substanzen zu bestimmen.

War dagegen das Reactionsproduct stickstoffhaltig, so konnte es entweder aus unverändertem Ausgangsmaterial oder

3) Vergl. Ber. d. deutsch. chem. Ges. 36, 1878 (1903) und 37, 1470 (1904). 
aus einem Gemisch von diesem mit einem Diphenylmethanderivat bestehen. Im ersteren Falle liess sich die Identität leicht feststellen; im letzteren musste das Gemisch zuvor zerlegt werden. Am zweckmässigsten hierfür erwies es sich, das Product in Benzol oder Aether aufzunehmen und aus dieser Lösung durch Chlorwasserstoff das salzsaure Salz der unveränderten Base auszufällen, das dann weiter untersucht wurde. Das Filtrat hinterliess beim Eindunsten die stickstofffreien Bestandtheile des Gemenges, also in erster Linie das Diphenylmethanderivat.

Diese Art der Untersuchung und Reinigung wurde auch vielfach dann angewandt, wenn die Probe auf Stickstoff mit Kalium negativ oder schwach ausgefallen war, da sie sicherer war und vor allem auch eine Schätzung der Menge noch vorhandener Ausgangssubstanz gestattete.

Ergänzt wurde die Untersuchung des Niederschlages durch die Prüfung des ursprünglichen Filtrates auf abgespaltetes Amin. $\mathrm{Zu}$ diesem Zweck machte man die Flüssigkeit durch Natronlange stark alkalisch und leitete einen Dampfstrom hindurch, bis alles eventuell abgespaltete Amin übergetrieben war. Das Destillat fing man entweder in $1 / 10 \mathrm{n}$-Salzsäure auf und bestimmte durch Titration die Menge der übergegangenen Base, oder man dampfte bei Anwendung gewöhnlicher Salzsäure das Destillat auf dem Wasserbade ein und zog aus der Menge des Rückstandes einen Schluss auf den Grad der Zersetzung. Ausserdem wurden die abgespaltenen Amine durch Platin- oder Quecksilbersalze identificirt. In Fällen, wo nur spurenweise eine Spaltung stattgefunden hatte, und dementsprechend beim Eindunsten des Destillates nur ein Anfling in der Schale zurückblieb, konnte die Anwesenheit der Base nur an dem Geruch erkannt werden, der beim Uebergiessen des Rückstandes mit Lauge auftrat.

\section{Verhalten gegen 100procentige Essigsäure.}

Die Einwirkung von Eisessig auf die Condensationsproducte verlief in viel mannigfaltigerer Weise, und demgemäss gestaltete 
sich auch die Aufarbeitung der Reactionsproducte vielfach schwieriger als bei den Versuchen mit Natronlauge.

Zunächst stellte sich heraus, dass sich schon ein Wassergehalt von $1-2 \mathrm{pC}$, wie er in dem gewöhnlichen ,Eisessig“" vorhanden zu sein pflegt, bei den Reactionen bemerkbar macht, da in einer Reihe von Fällen festgestellt werden konnte, dass gewöhnlicher Eisessig und reine Essigsäure wesentlich verschiedene Zersetzungsproducte liefern. Es wurde daher für alle Versuche eine Essigsäure verwendet, die höchstens $0,2-0,3 \mathrm{pC}$. Wasser enthielt.

Bei den Versuchen in der Kälte löste man in der Regel 0,5-1 g Substanz in soviel Essigsäure als erforderlich war und liess 24 Stunden verschlossen stehen; bei den anderen kochte man die gleiche Menge mit $5 \mathrm{ccm}$ Eisessig eine Stunde am Steigrohre uber freier Flamme. Manche Verbindungen konnten in der Kälte nicht in normaler Weise untersucht werden, da ihre Löslichkeit in kaltem Eisessig zu gering war.

Nicht weniger als die Beschaffenheit der Essigsäure übt auch die Verarbeitungsweise der Reactionsflüssigkeit einen EinHuss auf die Art der schliesslich entstehenden Producte aus. Beispielsweise scheidet sich aus essigsauren Lösungen der Verbindungen

und

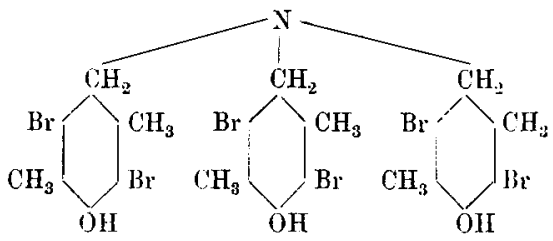<smiles>C[C@H](Br)[C@H](C)Br</smiles>

beim Erkalten und freiwilligen Verdunsten das Diphenylmethanderivat 


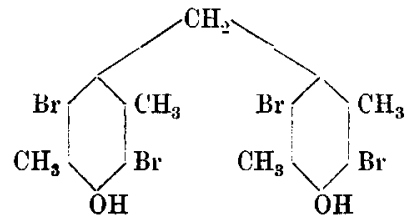

aus, während aus den gleichen Lösungen durch Wasser der Körper

gefällt wird.<smiles>CCOCCC(CBr)C(C)O</smiles>

Der Einheitlichkeit halber wurden die Reactionsproducte sämmtlicher Versuche in folgender Weise aufgearbeitet:

Hatten sich aus den Lösungen nach 24 stündigem Stehen oder nach dem Erkalten Krystalle in genügender Menge ausgeschieden, so wurden diese abfiltrirt und für sich untersucht. Das Filtrat oder, wenn nur eine geringe oder gar keine Ausscheidung stattgefunden hatte, die ursprüngliche Flüssigkeit verdünnte man mit Wasser und fügte zum Schlusse, um die fast stets eintretende, oft krystallinische Fällung zu vervollständigen, Sodalösung hinzu, bis die Reaction neutral oder eben noch schwach sauer war. Der Niederschlag wurde filtrirt, mit Wasser gewaschen und auf Thon getrocknet, das Filtrat nach Zusatz von Alkali in derselben Weise wie bei den Versuchen mit Natronlauge auf abgespaltenes Amin geprüft.

Waren die ursprüngliche Ausscheidung und die spätere Fällung frei von Stickstoff, so konnten in ihnen Diphenylmethanderivates I) oder Acetylverbindungen (II und III) des dem Condensationsproducte entsprechenden Phenolalkohols oder Gemische von diesen Substanzen vorliegen:

I.

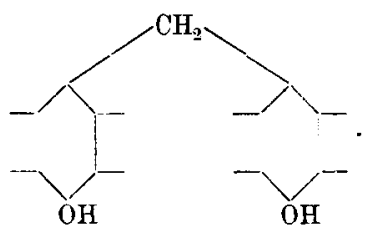


II.

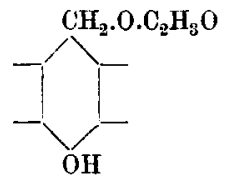

III.

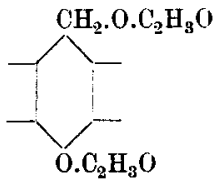

Verbindungen des Typus II und III sollen im Folgenden der Kürze halber stets als „Monoacetat" und „Diacetat" bezeichnet werden.

Handelte es sich um einheitliche Producte, so bot deren Erkennung keine Schwierigkeiten, da fast regelmässig Vergleichpräparate zur Verfügung standen, oder die Natur des Körpers durch eine Analyse festgestellt werden konnte.

Erwiesen sich die Substanzen als Gemische, so kochte man sie mit wenig Ligroïn aus, das die Diphenylmethankörper ungelöst zurückliess und die Acetate aufnahm. Umkrystallisiren des Rückstandes aus siedendem Benzol, der gelösten Verbindung aus Ligroïn und Petroläther führte dann in der Regel rasch zu reinen Präparaten.

Wenn dagegen die Ausscheidungen und Fällungen Stickstoff enthielten, so musste geprüft werden, ob man die unveränderte Base, bezw. deren normales Acetylirungsproduct oder Gemische dieser Substanzen mit einer oder mehreren der eben erwähnten stickstofffreien vor sich hatte.

Auch hier gelang die Identificirung in der Regel leicht, weun das Product einheitlich war; nöthigenfalls wurde die Acetylverbindung überdies durch Verseifung in das Ausgangsmaterial zurückverwandelt.

Schwieriger gestaltete sich die Aufarbeitung der Gemische, bei der gewöhnlich zunächst die basischen Bestandtheile durch Chlorwasserstoff aus benzolischer oder ätherischer Lösung ausgefällt wurden, während man den Verdunstungsrückstand des Filtrates entweder direct umkrystallisirte oder zuvor durch Behandlung mit Ligroïn weiter in seine Bestandtheile zerlegte. Bei complicirten Gemischen ist es jedoch nicht in allen Fällen gelungen, sämmtliche Spaltungsproducte mit Sicherheit zu er- 
kennen, da die einzelnen Versuche mit kleinen Substanzmengen angestellt wurden, und es nicht lohnend erschien, zur Entscheidung von Fragen untergeordneter Bedeutung grössere Quantitäten Materials aufzuwenden.

Nicht immer schieden sich die Reactionsproducte bei den Versuchen mit Essigsäure in fester Form aus; manche Producte blieben vielmehr auch bei tagelangem Stehen unter Wasser schmierig oder ölig. Man nahm dann die Substanzen in Aether auf, wusch und trocknete die Lösung und versuchte dann durch vorsichtiges Eindunsten, eventuell unter Zusatz von Ligroïn, zu festen Producten zu gelangen; oder man behandelte den ätherischen Auszug mit Salzsäuregas und verfuhr dann wie oben. Wenn nöthig, wurden diese Operationen auch mehrfach ausgeführt.

\section{Verhaltein gegen Essigsäureanhydrid.}

$1 \mathrm{~g}$ Substanz kochte man mit dem Anhydrid eine Stunde über freier Flamme unter Rückfluss, goss dann die Lösung in Wasser und fügte allmählich, um Erwärmung und Verseifung zu vermeideu, verdünnte Natronlauge hinzu, bis die Flüssigkeit nur noch schwach sauer reagirte. Sobald das Reactionsproduct völlig oder einigermassen erstarrt war, was häufig erst nach mehrtägigem Stehen eintrat, filtrirte man ab und untersuchte das alkalisch gemachte Filtrat in der angegebenen Weise auf Amin.

Stickstofffreie Spaltungsproducte erwiesen sich fast immer als "Diacetate" der Phenolalkohole, die sich meist leicht umkrystallisiren und mit Vergleichpräparaten identificiren liessen. Nur in seltenen Fällen entstanden die Acetylverbindungen von Diphenylmethanderivaten, die analysirt und durch Verseifung in ihre bekannten Stammkörper übergeführt wurden.

Stickstoffhaltige Reactionsproducte bestanden entweder aus den normalen Acetylderivaten der Condensationsproducte, die gleichfalls analysirt und, je nach ibrer Constitution, durch Kochen mit alkoholischer Lange vollständig oder partiell ver- 
seift wurdeu; oder sie stellten Gemenge von diesen Substanzen mit den eben besprochenen stickstofffreien Acetaten vor. Dann konnte in manchen Fällen durch Salzsäuregas eine Trennung bewirkt werden; in anderen erwies sich fractionirte Krystallisation als wirksamer; bestimmte Regeln liessen sich für die Verarbeitung dieser Gemische nicht aufstellen. Gelang eine Scheidung der Bestandtheile uberhaupt nicht, so verseifte man das Ganze und suchte dann die meist schwerer löslichen und besser krystallisirenden Verseifungsproducte nach den bekannten Methoden zu trennen.

Nicht selten war das Reactionsproduct überhaupt nicht zum Erstarren zu bringen. Dann wurde es entweder direct verseift oder zunächst in ätherischer Lösung mit Salzsäuregas behandelt, worauf dann die üblichen Trennungs- und Identificirungsversuche erfolgten.

Versuche mit Essigsäureanhydrid in der Kälte wurden nur vereinzelt angestellt.

\section{Verhalten auf dem Wasserbade.}

Eine Probe der Substanz wurde zwischen zwei Uhrgläsern, deren oberes innen einen feuchten Streifen rothen Lackmuspapiers trug, auf einem lebhaft siedenden Wasserbade erbitzt. Bläute sich der Streifen, so erneuerte man das Lackmuspapier von Zeit zu Zeit, um das Fortschreiten der Reaction beobachten zu können. Bei sehr zersetzlichen Substanzen wurde das Erhitzen auf wenige Stunden beschränkt, bei beständigeren bis zu drei Tagen, d. h. $3 \times 8$ bis 10 Stunden, ausgedehnt.

Hatte sich der Streifen nicht gebläut, so erwies sich regelmässig die Substanz nach Aussehen und Schmeizpunkt als unzersetzt. Im anderen Falle wurde zunächst geprüft, ob die zurückgebliebene Masse noch Stickstoff enthielt. Fehlte Stickstoff, oder war er nur noch in ganz geringer Menge vorhanden, so konnte das Product direct aus siedendem Benzol umkrystallisirt werden und wurde dann als ein Diphenylmethanderivat erkannt. War der Stickstoffgehalt beträchtlicher, so nahm man 
den Rückstand in Aether oder siedendem Benzol auf, entferute die unveränderte Base durch Einleiten von Chlorwasserstoff und prüfte, ob das Filtrat beim Eindunsten ein Diphenylmethanderivat hinterliess.

In den wenigen Fällen, in denen es sich um Abspaltung von Aminen handelte, die auf Lackmus nicht reagiren, konnte der Verlauf der Reaction natürlich nur durch eine genaue Untersuchung der auf dem Wasserbade verbliebenen Substanz festgestellt werden.

\section{Uebersicht über die Versuche.}

In der folgenden Zusammenstellung sind nur zum kleinen Theile die Resultate von Einzelversuchen verzeichnet; die meisten Angaben beziehen sich auf Reihen von zwei, drei oder mehr Versuchen, deren Ergebnisse abgerundet eingesetzt worden sind. Soweit nähere Angaben über den Verlauf der einzelnen Versuche nöthig erschienen, sind sie in den folgenden Arbeiten gegeben; austührlichere Beschreibungen finden sich zum Theil in den Dissertationen der betheiligten Herren. Alle Versuche mit stärkerer oder schwächerer Natronlauge, mit Alkoholen und aromatischen Kohlenwasserstofien, auch mit den Aethern und Estern der Basen, sind aus den Tabellen weggelassen worden; hierüber findet sich gleichfalls das Nöthige in den Specialarbeiten.

In den ersten vier Tabellen sind die Versuche mit den Derivaten der vier Basen zusammengestellt, mit denen die meisten der untersuchten Bromide condensirt worden sind. Die beiden folgenden enthalten die Versuche mit den Deriraten der übrigen Amine.

Die Tabelle VII giebt in noch mehr gekürzter Form die wesentlichen Ergebnisse sämmtlicher Versuche, damit man aus ihr gleichzeitig einen Ueberblick über den Einfluss der phenolischen und der basischen Componenten auf die Beständigkeit der verschiedenen Condensationsproducte gewinnen kann.

Zur Erläuterung der Tabellen sei noch Folgendes bemerkt: 
In den ersten sechs Tabellen sind unter a. die in der Kälte, unter b. die in der Hitze ausgeführten Versuche verzeichnet. In der Tabelle VII bezieht sich $1 \mathrm{a}$. und b. auf die Versuche mit Natronlauge, 2 a. und $b$. auf diejenigen mit Essigsäure, 3. auf die mit Essigsäureanhydrid und 4. auf die Versuche, bei denen die Substanzen auf dem Wasserbade erhitzt wurden.

Ferner bedeutet ein eingeklammertes (fast) oder (f.), dass nur Spuren von Amin abgespaltet worden sind, die lediglich am Geruche erkannt werden konnten. Der Ausdruck ,unzersetzt" besagt, dass eine Abspaltung von Amin nicht stattgefunden hat, das Condensationsproduct aber doch verändert, $d . b$. in diesen Fällen in sein normales Acetylirungsproduct umgewandelt worden ist ${ }^{4}$ ). Ein Fragezeichen hinter der Bezeichnung eines Reactionsproductes deutet an, dass eine Zersetzung eingetreten war und nach der Untersuchung sowie Analogien mit grosser Wahrscheinlichkeit auf das Vorliegen der betreffenden Substanz geschlossen werden konnte, dass aber eine bestimmte Identificirung entweder nicht gelungen, oder überhaupt nicht versucht worden ist. Wo sich nichts uber die Natur der Spaltungsproducte aussagen lässt, steht einfach ,,zersetzt" in den Tabellen.

4) Bei der Eiuwirkung von Eisessig auf die Benzylaminderivate der Form Ar. $\mathrm{CH}_{2} \cdot \mathrm{NH} \cdot \mathrm{C}_{7} \mathrm{H}_{7}$ entstanden vielfach wechselnde Gemische von unveränderter Base und deren normalem Acetylirungsproduct. In den Tabellen ist der Einfachheit halber in diesen Fällen die Bezeichnung ,unverändert" eingesetzt worden, da der Unterschied belanglos ist. 


\section{Tabelle $I$.}

Methylaminderivate.

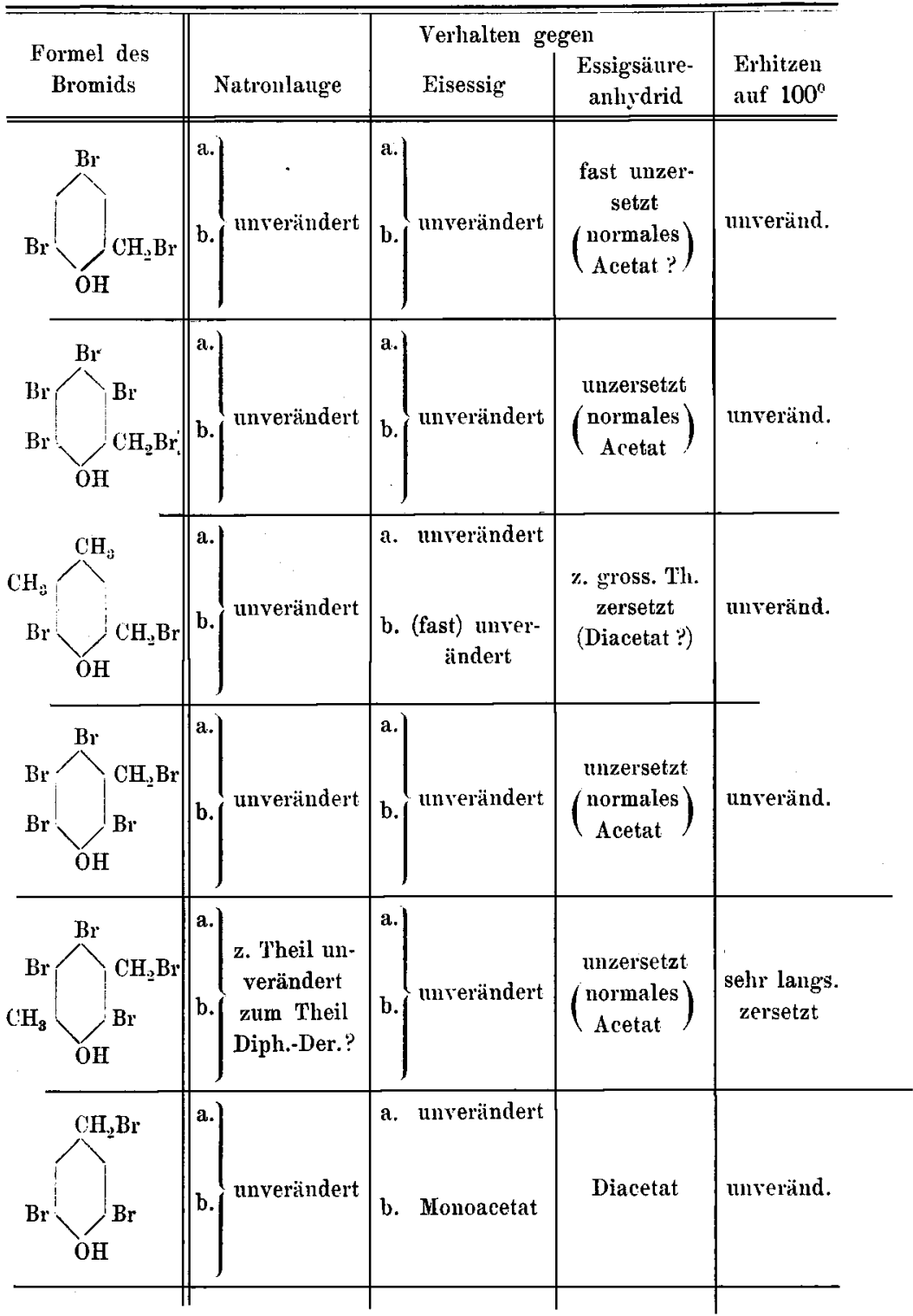




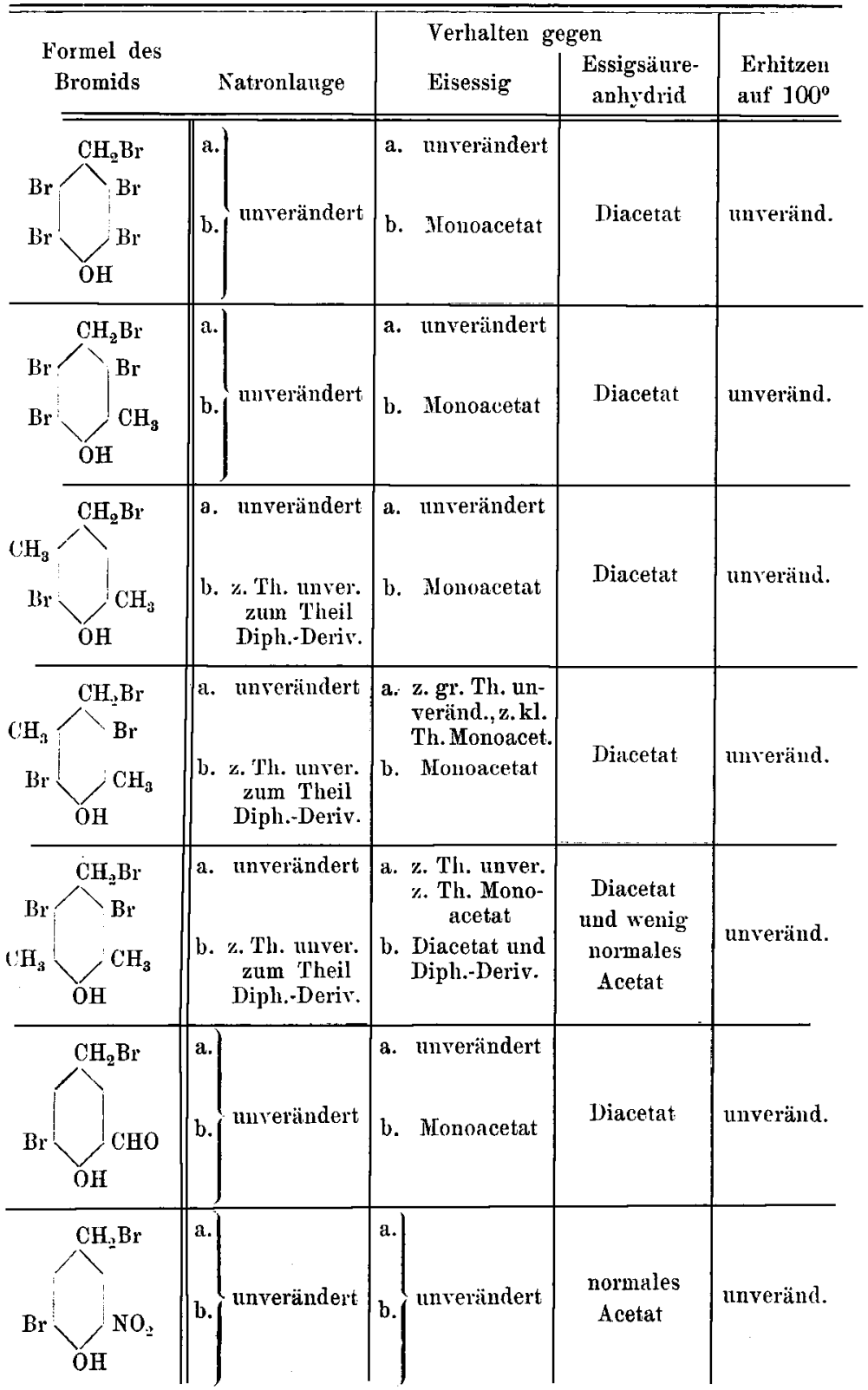




\section{Tabelle II.}

\section{Piperidinderivate.}

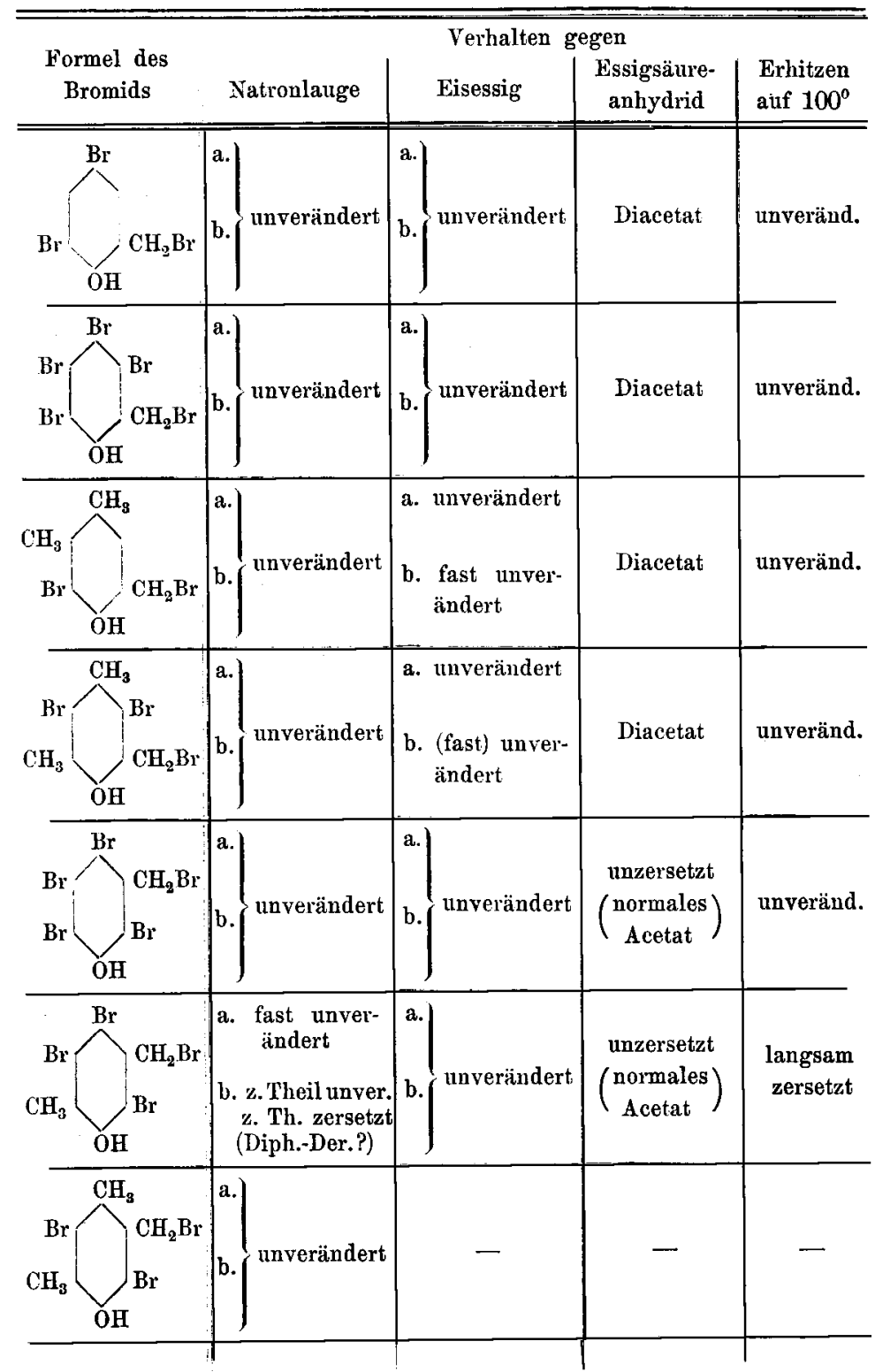




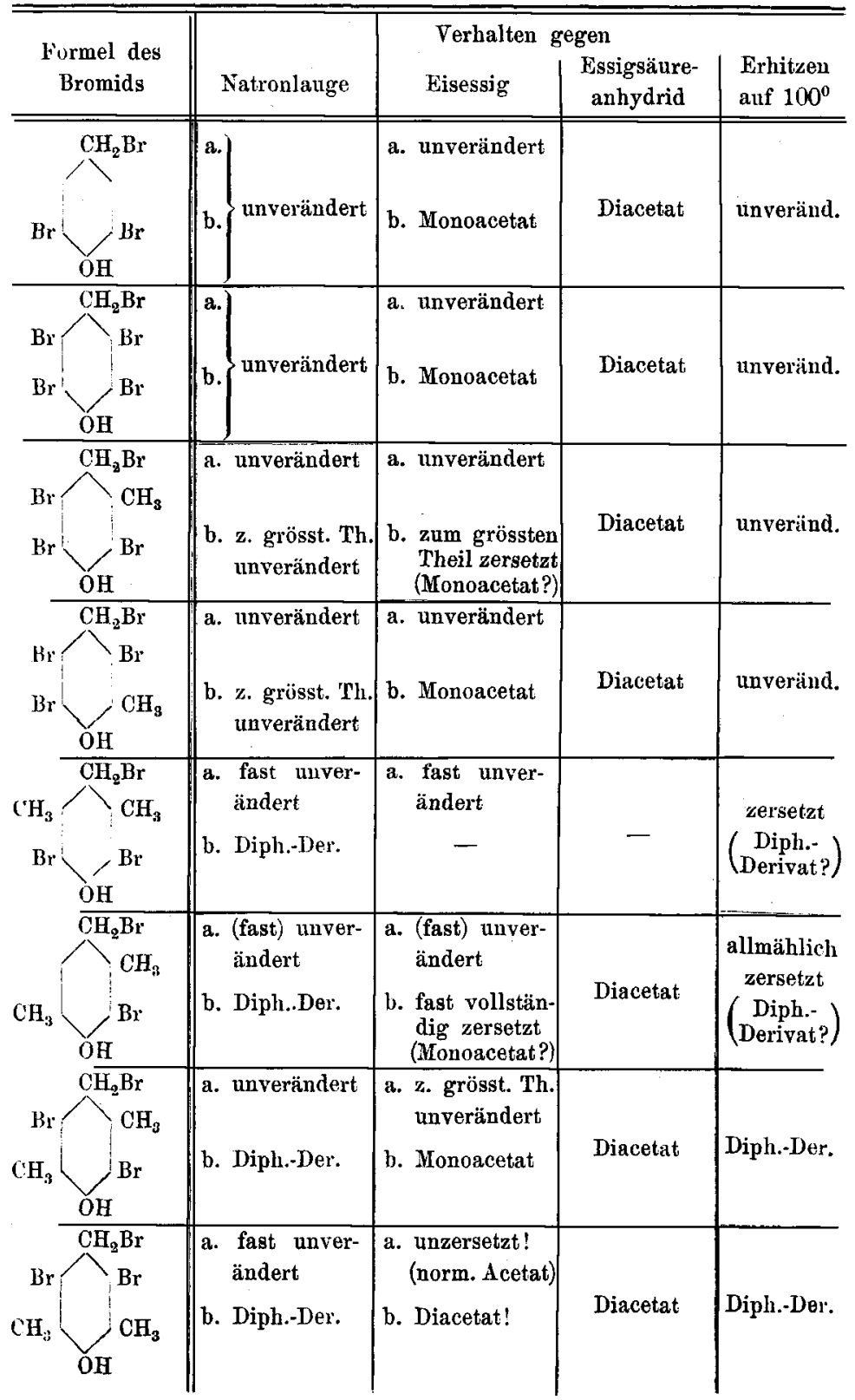




\section{Tabelle III.}

\section{Benzylaminderivate}

von der Form R.CH $\cdot$ NH. $\mathrm{CH}_{2} \cdot \mathrm{C}_{6} \mathrm{H}_{5}$.

\begin{tabular}{|c|c|c|c|c|}
\hline \multirow{2}{*}{$\begin{array}{l}\text { Formel des } \\
\text { Bromids }\end{array}$} & \multicolumn{4}{|c|}{ Verhalten gegen } \\
\hline & Natronlauge & Eisessig & $\begin{array}{c}\text { Essigsäure- } \\
\text { anhydrid }\end{array}$ & $\begin{array}{l}\text { Erhitzen } \\
\text { auf } 100^{\circ}\end{array}$ \\
\hline $\mathrm{CH}_{2} \mathrm{Br}$ & b. unverändert & $\begin{array}{l}\text { a. unverändert } \\
\text { b. z. grösst. Th. } \\
\text { unverändert }\end{array}$ & $\begin{array}{c}\text { z. grösst. Th. } \\
\text { unzersetzt } \\
\left(\begin{array}{c}\text { normales } \\
\text { Acetat }\end{array}\right)\end{array}$ & imveränd. \\
\hline $\begin{array}{l}\mathrm{Br} \\
\mathrm{CH}_{2} \mathrm{Br}\end{array}$ & a. $\left\{\begin{array}{l}\text { z. grösst. Th. } \\
\text { b. }\end{array}\right.$ & $\begin{array}{l}\text { a. z. grösst. Th. } \\
\text { unverändert } \\
\text { b. z. grösst. Th. } \\
\text { Monoacetat }\end{array}$ & $\begin{array}{l}\text { z. gröss. Th. } \\
\text { unzersetzt } \\
\left.\text { ( } \begin{array}{c}\text { normales } \\
\text { Acetat }\end{array}\right)\end{array}$ & unveränd. \\
\hline$\overbrace{\mathrm{OH}}^{\mathrm{Br}}$ & $\begin{array}{l}\text { a. unverändert } \\
\text { b. fast unver- } \\
\text { ändert }\end{array}$ & $\begin{array}{l}\text { a. fast unver- } \\
\text { ändert } \\
\text { b. Diph.-Deriv. }\end{array}$ & $\begin{array}{c}\text { unzersetzt } \\
\left(\begin{array}{c}\text { normales } \\
\text { Acetat }\end{array}\right)\end{array}$ & 一 \\
\hline $\int_{\mathrm{Br}}^{\mathrm{Br}}$ & b. & $\begin{array}{l}\text { a. unverändert } \\
\text { b. etwa } 3 / 5 \text { Diph.- } \\
\text { Der., etwa } 2 / 5 \\
\text { Monoacet. }\end{array}$ & $\begin{array}{c}\text { unzersetzt } \\
\left(\begin{array}{c}\text { normales } \\
\text { Acetat }\end{array}\right)\end{array}$ & nuveränd. \\
\hline$\left\{\begin{array}{l}\mathrm{Br} \\
\mathrm{CH}_{3} \mathrm{Br}\end{array}\right.$ & b. unverändert & $\begin{array}{l}\text { a. unverändert } \\
\text { b. Monoacetat }\end{array}$ & $\begin{array}{c}\text { unzersetzt } \\
\left(\begin{array}{c}\text { normales } \\
\text { Acetat }\end{array}\right)\end{array}$ & unveränd. \\
\hline$\overbrace{\mathrm{OH}}^{\mathrm{Br}}$ & $\begin{array}{l}\text { a. unverändert } \\
\text { b. z. grösst. Th. } \\
\text { unver., wenig } \\
\text { Diph.-Deriv. }\end{array}$ & $\begin{array}{l}\text { a. z. grösst. Th. } \\
\text { unverändert } \\
\text { b. z. gr. Th.zers., } \\
\left(\begin{array}{l}\text { Acetate } \\
\text { u. Diph.-Der. })\end{array}\right.\end{array}$ & $\begin{array}{c}\text { uuzersetzt } \\
\left(\begin{array}{c}\text { normales } \\
\text { Acetat }\end{array}\right)\end{array}$ & $\begin{array}{c}\text { fast } \\
\text { unveränd. }\end{array}$ \\
\hline
\end{tabular}


mit substituirten Oxybenzylbromiden.

Benzylaminderivate

von der Form

R. $\mathrm{CH}_{2} \cdot \mathrm{N} \cdot \mathrm{CH}_{2} \cdot \mathrm{R}$

$$
\mathrm{CH}_{2} \cdot \mathrm{C}_{6} \mathrm{H}_{5}
$$

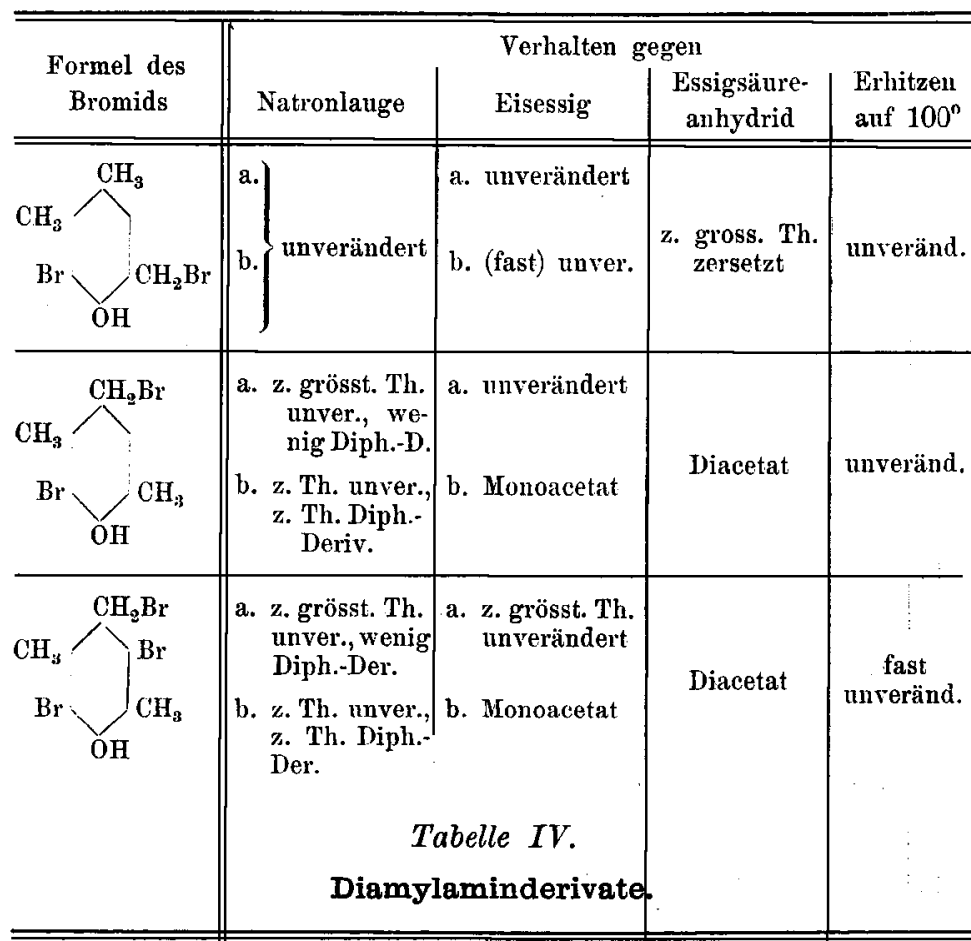

\begin{tabular}{|c|c|c|c|c|}
\hline $\begin{array}{c}\text { Formel des } \\
\text { Bromids }\end{array}$ & Natronlaug'e & $\begin{array}{l}\text { Verhalten } \\
\text { Eisessig }\end{array}$ & $\begin{array}{l}\text { Essigsäure- } \\
\text { anhydrid }\end{array}$ & $\begin{array}{l}\text { Erhitzen } \\
\text { auf } 100^{\circ}\end{array}$ \\
\hline $\mathrm{OH}^{\mathrm{OH} B r}$ & b. $\left\{\begin{array}{l}\text { unvösst. Th. Th. } \\
\text { undert }\end{array}\right.$ & $\begin{array}{l}\text { a. unverändert } \\
\text { b. z. grösst. Th. } \\
\text { Monoacetat }\end{array}$ & $\begin{array}{c}\text { zersetzt } \\
\text { (Diacetat?) }\end{array}$ & unverïnd. \\
\hline $\mathrm{Br}_{\mathrm{Br}}^{\mathrm{Br}} \mathrm{CH}_{\mathrm{n}} \mathrm{Br}$ & $\begin{array}{l}\text { a. } \\
\text { b. grösst. Th. } \\
\text { znverändert }\end{array}$ & $\begin{array}{l}\text { a. z. grösst. Th. } \\
\text { unverändert } \\
\text { b. Monoacetat }\end{array}$ & $\begin{array}{c}\text { zersetzt } \\
\text { (Diacetat?) }\end{array}$ & ınveränd. \\
\hline
\end{tabular}




\begin{tabular}{|c|c|c|c|c|}
\hline \multirow[b]{2}{*}{$\begin{array}{c}\text { Formel des } \\
\text { Bromids }\end{array}$} & \multicolumn{4}{|c|}{ Verhalten gegen } \\
\hline & Natronlatuge & Eisessig & $\begin{array}{c}\text { Essigsäure- } \\
\text { anhydrid }\end{array}$ & $\begin{array}{l}\text { Erhitzen } \\
\text { auf } 100^{\circ}\end{array}$ \\
\hline $\begin{array}{r}\mathrm{CH}_{3} \\
\mathrm{Br}\end{array}$ & - & $\begin{array}{l}\text { a. z. Th. unver. } \\
\text { z.Th. zersetzt } \\
\text { b. zersetzt }\end{array}$ & Diacetat & - \\
\hline $\begin{array}{l}\mathrm{CH}_{2} \mathrm{Br} \\
\mathrm{Br}\end{array}$ & b. $\{$ unverändert & $\begin{array}{l}\text { a. unverändert } \\
\text { b. fast unver. }\end{array}$ & $\begin{array}{c}\text { unzersetzt } \\
\left(\begin{array}{c}\text { normales } \\
\text { Acetat }\end{array}\right)\end{array}$ & unveränd. \\
\hline $\begin{array}{l}\mathrm{CH}_{2} \mathrm{Br} \\
\mathrm{Br}\end{array}$ & 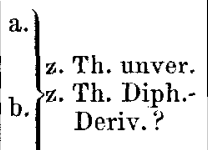 & $\begin{array}{l}\text { a. unverändert } \\
\text { b. Diph.-Der.? }\end{array}$ & $\left(\begin{array}{c}\text { zersetzt } \\
\text { Acetat des } \\
\text { Diph.-Der.? }\end{array}\right)$ & $\left(\begin{array}{c}\text { zersetzt } \\
\text { Diph.- } \\
\text { Derivat? }\end{array}\right)$ \\
\hline $\mathrm{CH}_{2} \mathrm{Br}$ & $\begin{array}{l}\text { a. fast unver. } \\
\text { b. etwa } 3 / 4 \text { un- } \\
\text { ver., etwa } 1 / 4 \\
\text { zersetzt }\end{array}$ & $\begin{array}{l}\text { a. fast unver. } \\
\text { b. Diph.-Der. }\end{array}$ & $\left(\begin{array}{c}\text { zersetzt } \\
\text { Acetat des } \\
\text { Diph.-Der.? }\end{array}\right)$ & $\begin{array}{c}\text { allmählich } \\
\text { zersetzt }\end{array}$ \\
\hline $\int_{\mathrm{Br}}^{\mathrm{CH}}$ & $\begin{array}{l}\text { a. z. grösst. Th. } \\
\text { unverändert } \\
\text { b. Diph.-Der.! }\end{array}$ & $\left.\left.\right|_{\text {b. }} ^{\text {a. }}\right|_{\text {Diph.-Der.! }}$ & $\begin{array}{c}\text { zersetzt } \\
\left(\begin{array}{l}\text { Acetat des } \\
\text { Dipl.-Der. }\end{array}\right)\end{array}$ & $\begin{array}{c}\text { allmählich } \\
\text { zersetzt }\end{array}$ \\
\hline $\begin{array}{l}\mathrm{CH}_{\mathrm{a}} \mathrm{Br} \\
\mathrm{Br} \\
\mathrm{CH}_{3}\end{array}$ & $\begin{array}{l}\text { a. unverändert } \\
\text { b. z. grösst. Th. } \\
\text { unverändert }\end{array}$ & $\begin{array}{l}\text { a. z. grösst. Th. } \\
\text { unverändert } \\
\text { b. Monoacetat }\end{array}$ & Diacetat & $\begin{array}{l}\text { allmählich } \\
\text { zersetzt }\end{array}$ \\
\hline
\end{tabular}




\begin{tabular}{|c|c|c|c|c|}
\hline N & \multicolumn{4}{|c|}{ Verhalten gegen } \\
\hline $\begin{array}{l}\text { Formel des } \\
\text { Bromids }\end{array}$ & Natronlauge & Eisessig & $\begin{array}{c}\text { Essigsäure- } \\
\text { anhydrid }\end{array}$ & $\begin{array}{l}\text { Erhitzen } \\
\text { auf } 100^{\circ}\end{array}$ \\
\hline $\int_{\mathrm{Br}}^{\mathrm{CH}_{3}}$ & b. & - & - & zersetzt \\
\hline $\mathrm{Br}$ & $\begin{array}{l}\text { a. z. gross. Th. } \\
\text { unver., z. k1. } \\
\text { 'Th.Diph.-Der. } \\
\text { b. Diph.-Deriv. }\end{array}$ & $\begin{array}{l}\text { a. etwa 1/2 zer- } \\
\text { setzt } \\
\text { b. zersetzt } \\
\text { (Monoacetat?) }\end{array}$ & Diacetat & $\begin{array}{c}\text { raseh } \\
\text { zersetzt } \\
\text { (Diph.-Der.) }\end{array}$ \\
\hline$\underbrace{\mathrm{CH}_{2} \mathrm{Br}}_{\mathrm{CH}_{3}}$ & $\begin{array}{c}\text { a. etwa }{ }^{1 / 2} \text { unver. } \\
\text { Derivat } \\
\text { b. Diph.-Deriv. }\end{array}$ & $\begin{array}{c}\text { a. etwa }{ }^{1 / 3} \text { unver. } \\
{ }^{2 / 3} \text { Diph.- } \\
\text { Derivat } \\
\text { b. Monoacetat }\end{array}$ & Diacetat & $\begin{array}{c}\text { rasch } \\
\text { zersetzt } \\
\text { (Diph.-Der. }\end{array}$ \\
\hline $\begin{array}{l}\mathrm{Br} \\
\mathrm{CH}_{3}\end{array}$ & $\begin{array}{l}\text { a. etwa }{ }^{2} / 3 \text { unver. } \\
1 / 3 \text { Diph.- } \\
\text { Derivat } \\
\text { b. Diph.-Deriv. }\end{array}$ & $\begin{array}{l}\text { a. z. grösst. Th. } \\
\text { Diph.-Der. } \\
\text { b. Diacetat }\end{array}$ & $\begin{array}{c}\text { Diacetat } \\
\text { und } \\
\text { Acetat des } \\
\text { Diph.-Deriv. }\end{array}$ & $\begin{array}{c}\text { rasch } \\
\text { zersetzt } \\
\text { (Diph.-Der.) }\end{array}$ \\
\hline $\mathrm{CH}_{2} \mathrm{Br}$ & $\left.\right|_{\text {b. }} ^{\text {a. }}$ & $\begin{array}{l}\text { a. unverändert } \\
\text { b. Monoacetat }\end{array}$ & Monoacetat? & $\begin{array}{l}\text { sehr lang- } \\
\text { sam zersetzt }\end{array}$ \\
\hline $\mathrm{CH}_{2} \mathrm{Br}$ & b. & $\begin{array}{l}\text { a. nnverändert } \\
\text { b. Monoacetat }\end{array}$ & Diacetat & unveränd. \\
\hline & & & & \\
\hline
\end{tabular}


Tabelle $\nabla$.

Ammoniakderivate.

\begin{tabular}{|c|c|c|c|c|}
\hline \multirow{2}{*}{$\begin{array}{c}\text { Formel des } \\
\text { Bromids }\end{array}$} & \multicolumn{4}{|c|}{ Verhalten gegen } \\
\hline & Natronlauge & Eisessig & $\begin{array}{c}\text { Essigsäure- } \\
\text { anbydrid }\end{array}$ & $\begin{array}{l}\text { Erhitzen } \\
\text { auf } 100^{\circ}\end{array}$ \\
\hline $\mathrm{OH}^{\mathrm{Br}}$ & $\begin{array}{l}\text { a. unverïndert } \\
\text { b. z. grösst. Th. } \\
\text { unver., wenig } \\
\text { Diph.-Der. }\end{array}$ & $\begin{array}{l}\text { a. } \quad- \\
\text { b. Monoacetat } \\
\text { oder } \\
\text { Diph.-Der. }\end{array}$ & Diacetat & unveränd. \\
\hline$\overbrace{\mathrm{OH}}^{\mathrm{Br}}$ & b. unverändert & $\begin{array}{l}\text { a. unverändert } \\
\text { b. z. grösst. Th. } \\
\text { unverändert }\end{array}$ & $\left.\begin{array}{c}\text { unzersetzt } \\
\text { normales } \\
\text { Acetat }\end{array}\right)$ & unveränd. \\
\hline $\int_{\mathrm{OH}}^{\mathrm{Br}} \mathrm{CH}_{3}$ & $\begin{array}{l}\text { Aethy] } \\
\text { a. z. Th. unver- } \\
\text { ändert, z. Th. } \\
\text { Diph.-Der. } \\
\text { b. Diph.-Der. }\end{array}$ & $\begin{array}{l}\text { aminderivate } \\
\text { a. z, Th. unver. } \\
\text { z. Th. Monoac. } \\
\text { b. z. grösst. Th. } \\
\text { Honoacetat }\end{array}$ & Diacetat & unveränd. \\
\hline $\begin{array}{l}\mathrm{CH}_{2} \mathrm{Br} \\
\mathrm{Br} \\
\mathrm{CH}_{3}\end{array}$ & $\begin{array}{l}\text { a. z. grösst. Th. } \\
\text { Diph.-Der. } \\
\text { b. Diph.-Der. } \\
\quad \rho \text {-Naph }\end{array}$ & $\begin{array}{l}\text { a. z. grösst. Th. } \\
\text { Monoacetat } \\
\text { u. Diph.-Der. } \\
\text { b. Diacetat und } \\
\text { Diph.-Der. } \\
\text { ylaminderiva }\end{array}$ & $\begin{array}{l}\text { Diacetat } \\
\text { te. }\end{array}$ & Diph.-Der. \\
\hline $\mathrm{OH}_{\mathrm{Or}}^{\mathrm{CH}_{3}}$ & b. $\int_{\text {unverändert }}^{\text {a. }}$ & $\begin{array}{l}\text { a. unverändert } \\
\text { b. unzersetzt } \\
\left(\begin{array}{l}\text { normales } \\
\text { N-Acetat }\end{array}\right)\end{array}$ & $\left(\begin{array}{c}\text { unzersetzt } \\
\text { O,N-Diacet. }\end{array}\right)$ & unveränd. \\
\hline $\begin{array}{l}\mathrm{CH}_{3} \mathrm{Br} \\
\mathrm{Br}\end{array}$ & b. unverändert & $\begin{array}{l}\text { a. unverändert } \\
\text { b. unzersetzt } \\
\left(\begin{array}{l}\text { normales } \\
\mathrm{N} \text {-Acetat }\end{array}\right)\end{array}$ & $\left(\begin{array}{c}\text { unzersetzt } \\
\text { normales } \\
\text { O,N-Diacet. }\end{array}\right)$ & unveränd. \\
\hline
\end{tabular}


mit substituirten Oxybenzylbromiden.

Anilinderivate.

\begin{tabular}{|c|c|c|c|}
\hline $\begin{array}{l}\text { Formel des } \\
\text { Bromids }\end{array}$ & Natronlauge & $\begin{array}{c}\text { Verhalten gegen } \\
\\
\text { Eisessig } \\
\text { Essigsäure- } \\
\text { anhydrid }\end{array}$ & $\begin{array}{l}\text { Errhitzen } \\
\text { auf } 100^{\circ}\end{array}$ \\
\hline $\mathrm{OH}_{\mathrm{OH}}^{\mathrm{Br}}$ & $\begin{array}{l}\text { a. unveründert } \\
\text { b. z. Th. unver. } \\
\text { z. Th. zersetzt }\end{array}$ & b. $\left(\begin{array}{cc}\text { unzersetzt } \\
\text { unzersetzt } \\
\text { n-Acetat }\end{array}\right):\left(\begin{array}{c}\text { normales } \\
O, N \text {-Diacet. }\end{array}\right)$ & nnveränd. \\
\hline $\int_{\mathrm{OH}}^{\mathrm{Br}} \mathrm{CH}_{3}$ & $\begin{array}{l}\text { a. unverändert } \\
\text { b. z. Th. unver. } \\
\text { z.Th. zersetzt }\end{array}$ & $\begin{array}{l}\text { a. unzersetyt } \\
\text { b. fast unzersetzt }\left(\begin{array}{c}\text { normales } \\
0, N \text {-Diacet. }\end{array}\right)\end{array}$ & $\begin{array}{c}\text { sehr langs. } \\
\text { zersetzt }\end{array}$ \\
\hline
\end{tabular}

Tabelle VI.

Diäthylaminderivate.

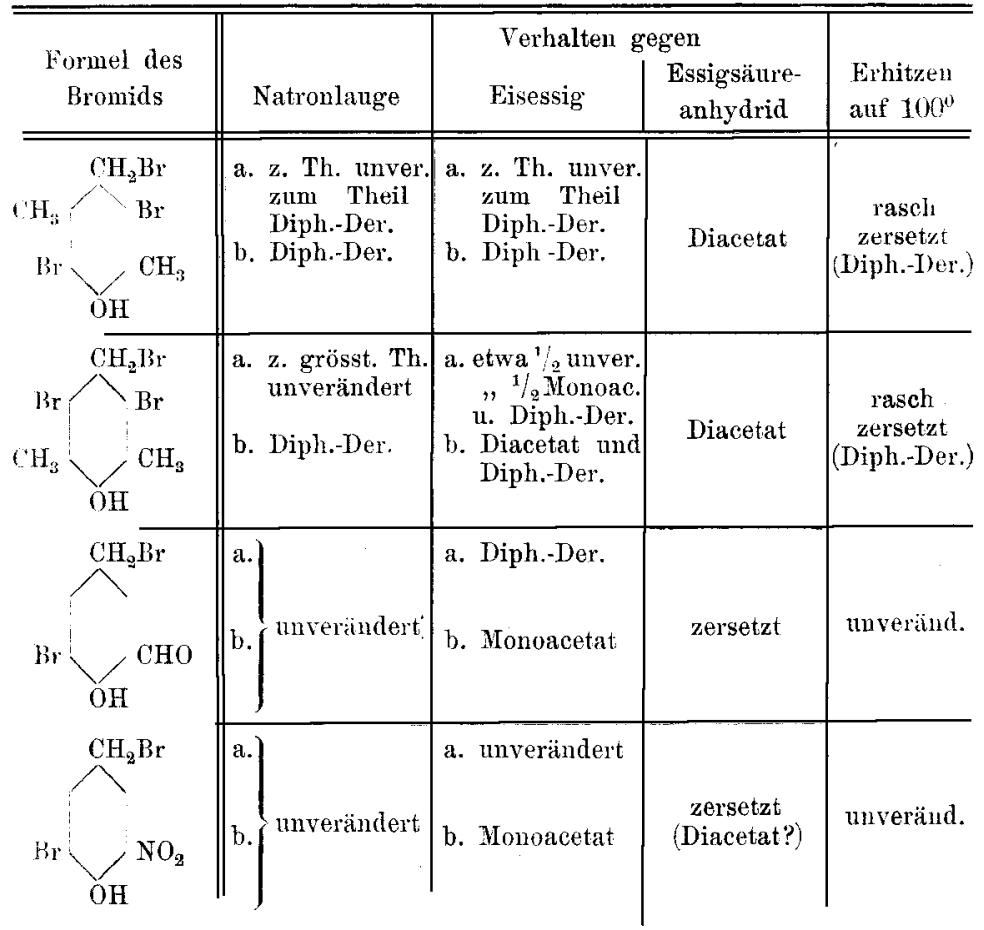


Methylanilinderivate.

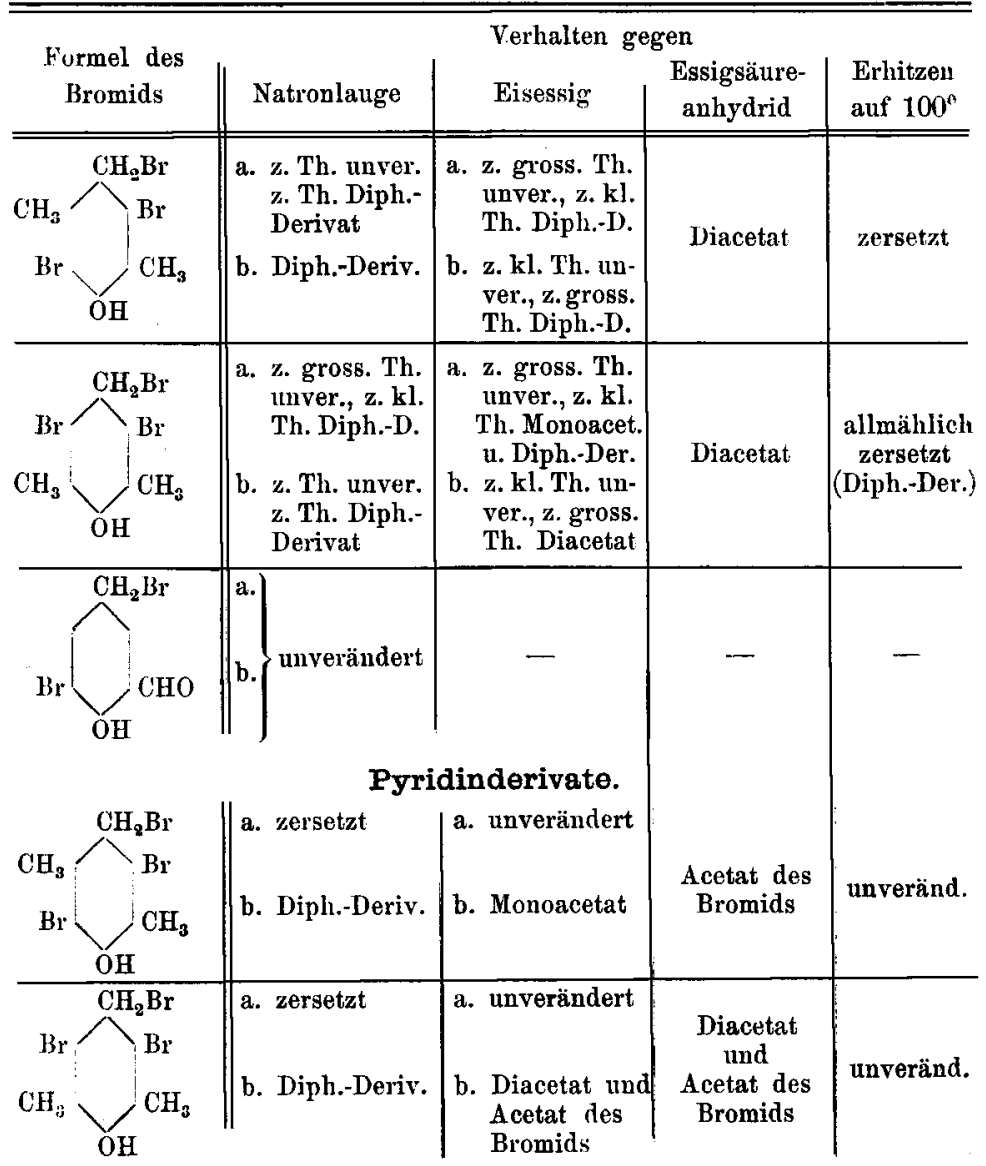

\section{Chinolinderivate.}

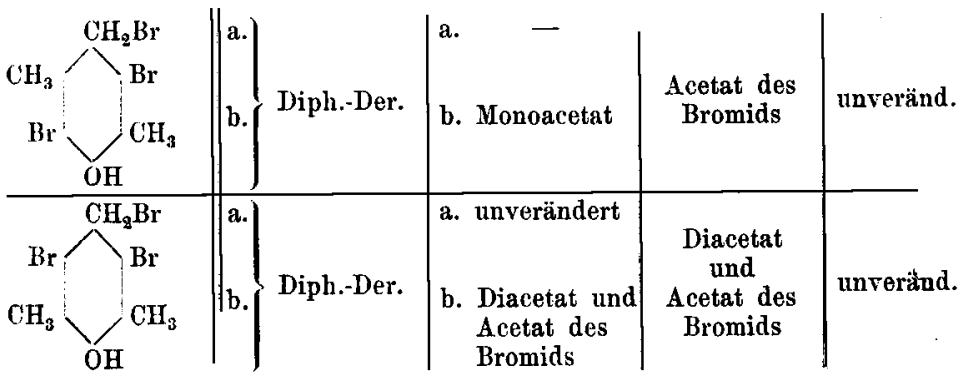




\section{Ergebnisse.}

Die mitgetheilten Versuche besitzen im Wesentlichen orientirenden Charakter und sollen in erster Linie eine allgemeine Kenntniss der in Betracht kommenden Verbältnisse vermitteln, sowie eine Vorarbeit für gegebenenfalls später auszuführende exacte Messungen bilden. Es könnte scheinen, als ob für diesen Zweck die Untersuchung unnöthig weit ausgedehnt worden sei. Auf Grund der Erfahrungen, die ich bei dem Studium der Pseudophenole und ihrer Umwandlungsproducte bisher gesammelt habe, ist jedoch eher das Gegentheil der Fall. Denn immer von Neuem wurde die Beobachtung gemacht, dass ihrer Constitution nach nahe verwandte Verbindungen bald bei dieser, bald bei jener Reaction ein überraschend verschiedenes Verhalten zeigten. Nur auf Grund eines sehr umfangreichen Beobachtungsmaterials kann man daher einigermassen sichere Schlüsse über den Einfluss der zahlreichen Factoren, die hier ins Spiel kommen, ziehen, während vereinzelte Beobachtungen nur einen geringen Werth besitzen. Es wäre daher sehr erwünscht gewesen, nicht nur mehr Combinationen zwischen den erwähnten Bromiden und Basen zu untersuchen, sondern vor allem auch noch einige weitere Pseudophenole von bestimmter Constitution, sowie ein paar andere Basen in den Kreis der Untersuchung zu ziehen. Die allzu grossen Schwierigkeiten, die sich der Gewinnung der nöthigen Materialien entgegenstellten, nicht zum Wenigsten auch die hohen Kosten, haben die Erfullung dieses Wunsches vorläufig verhindert, doch sind zur Zeit noch Versuche im Gange, um wenigstens einige der unerledigten Fragen zu lösen.

Noch fühlbarer als die Lücken, die in verschiedenen Beziehungen die Arbeit aufweist, macht sich der Umstand geltend, dass die Versuche überwiegend einen rein qualitativen Charakter tragen. Zu Beginn der Untersuchung wurden vorwiegend Verbindungen geprüft, die bei den einzelnen Reactionen entweder vollständig zersetzt wurden oder ganz unverändert 
blieben. Hier lag zunächst kein Anlass vor, etwa den Versuch zu machen, die Geschwindigkeit der verschiedenen Zersetzungen zu messen. Als sich dann die Zahl der Versuche mehrte, bei denen partielle Zersetzungen beobachtet wurden, trat allerdings der Wunsch nach genaueren Bestimmungen auf, doch musste aus praktischen Gründen einstweilen daranf verzichtet werden. Es stellte sich nämlich heraus, dass kleine Abweichungen in den Versuchsbedingungen, wie sie sich ohne Anwendung umständlicher Vorsichtsmaassregeln kaum vermeiden lassen, in vielen Fällen den Verlauf der Zersetzung so stark beeinflussen, dass die zahlenmässige Bestimmung des Zersetzungsgrades bei den in einfacher Form ausgeführten Versuchen zwecklos erschien. Die Durchführung der einzelnen Spaltungsversuche unter Bedingungen, die eine Sicherheit der Resultate bis auf wenige Procente gewährleistet hätte, würde einen solchen Aufwand an Zeit und Material erfordert haben, dass die Untersuchung auf eine sehr erheblich geringere Zahl von Verbindungen hätte beschränkt werden müssen; damit wäre aber der allgemeine Ueberblick, der zunächst erstrebt werden musste, verloren gegangen.

In den vorstehenden Tabellen ist daher, abgesehen von wevigen Fällen, von zablenmässigen Angaben abgesehen worden, obwohl eine Reihe von Versuchen quantitativ durchgeführt worden ist. Wo die Augaben verschiedener Beobachter erheblicher von einander abwichen, ist den von Herrn Dr. O. Schröter gewonnenen Ergebnissen grösseres Gewicht beigelegt worden, da er die ausgedehnteste Erfahrung besass und bei seinen Versuchen die innerhalb der angedeuteten Grenzen erreichbare Gleichförmigkeit der Ausfübrung am meisten gewahrt ist.

Nur eine Serie quantitativer Bestimmungen sei hier mitgetheilt, um wenigsteus einen Anhalt für eine etwas genauere Vergleichung der Zersetzlichkeit von Condensationsproducten verschiedener Bromide $z u$ bieten. Sie bezieht sich auf das Verhalten von 15 Piperidinderivaten gegen kochende fünfprocentige Natronlauge. 
Bei diesen Versuchen wurde je 0,5 $\mathrm{g}$ der verschiedenen Basen auf der analytischen Wage abgewogen, mit Alkohol quantitativ in einen Rundkolben gespült und mit $5 \mathrm{ccm}$ fünfprocentiger Natronlauge versetzt. Man verband dann den Kolben mit einem absteigenden Kühler, leitete eine Stunde lang einen möglichst gleichförmigen Strom von Wasserdampf hindurch, fing das übergehende Piperidin in einer gemessenen Menge $1 / 10 \mathrm{n}$ Salzsäure auf, titrirte zum Schluss mit $1 / 10 \mathrm{n}$-Barytwasser und Phenolphtaleïn zurück und berechnete aus der Menge der verbrauchten Salzsäure, wieviel Procente der angewandten Base zerstört worden warén.

Der Kürze halber sei voll der Wiedergabe der einzelnen experimentellen Daten abgesehen. In der umstehenden Tabelle VIII sind daher nur die Versuchsergebnisse in Procenten zersetzter Base angegeben. Die mitgetheilten Versuche sind sämmtlich von demselben Beobachter - Dr. O. Scbröter - ausgefübrt worden und stimmen bei den mässig zersctzlichen Verbindungen, wie ersichtlich, gut unter einander überein, während bei den Versuchen mit besonders unbeständigen Substanzen grosse Schwankungen auftreten, deren Ursachen hier nicht erörtert werden sollen.

Wie schon im Eingange erwähnt wurde, hängt die relative Beständigkeit der untersuchten Basen sowohl von der Natur des Aminrestes, als auch ron der des Phenolrestes ab.

In ersterer Beziehung lassen sich aus dem vorliegenden Beobachtungsmaterial bestimmte Gesetzmässigkeiten nicht ableiten; wohl aber kann man eine Anzahl empirischer Regeln aufstellen.

Von allen untersuchten Verbindungen haben sich die Derivate des $\beta$-Naphtylamins als die beständigsten erwiesen, denn sie werden von keinem der angewandten Agentien auch nur im Geringsten zersetzt. Zwar sind bisher nur die Combinationen dieses Amins mit den Tribromderivaten des Pseudocumenols und Mesitols geprüft worden, aber da die aus diesen beiden Pseudophenolen entstehenden Basen sich im Allgemeinen 
124 Auwers, Condensationsproducte organischer Basen

Tabelle VIII.

\section{Ortho-Verbindungen.}

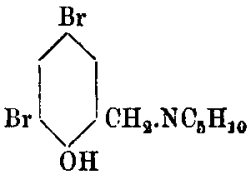

0<smiles>CCCCCC(O)C(Br)Br</smiles>

0<smiles>CCCCCCC(O)C(Br)CBr</smiles>

0<smiles>CCCCCCCCCCCCCCC</smiles>

0

Meta-Verbindungen.<smiles>CCCCCCCC(Br)Br</smiles>

0<smiles>C[AsH2]CC(Br)CBr</smiles>

24,8

$\frac{22,3}{\mathbf{2 8 , 5}}$<smiles>CCCCCCC(C)Br</smiles>

0

Para-Verbindungen.
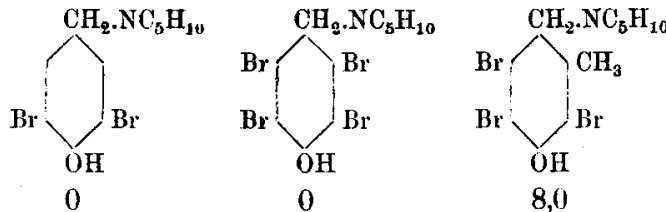<smiles>CCCCCC(Br)C(C)O</smiles>

$$
\begin{array}{r}
8,0 \\
10,6 \\
9,7 \\
8,0 \\
\hline \mathbf{9}
\end{array}
$$

12,4

15,9

12,4

$\frac{12,4}{13}$

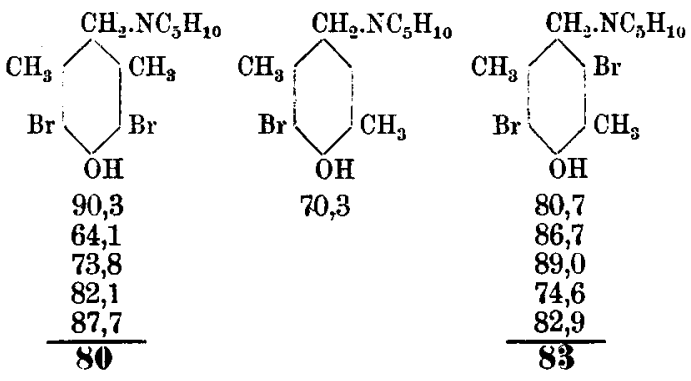


durch eine besonders hohe Zersetzlichkeit auszeichnen, kann man unbedenklich annehmen, dass die $\beta$-Naphtylaminderivate auch der anderen Bromide mindestens ebenso beständig sind.

Der nahe licgende Schluss, dass auch $\alpha$-Naphtylamin mit Pseudophenolen ztl stabilen Verbindungen zusammentritt, ist dagegen unzutreffend; diese Körper scheinen vielmehr nach den wenigen Versuchen, die in dieser Richtung angestellt worden sind, besonders empfindlich zu sein.

An das $\beta$-Naphtylamin reiht sich das Anilin an. Allerdings sind die Abkömmlinge dieses Amins nicht so bestăndig, wie früher angenommen wurde, denn in der Siedehitze werden sie durch verdunnte Laugen beträchtlich angegriffen und spalten sogar bei andauerndem Kochen im Dampfstrome das Amin ab. Und für die Derivate der Homologen des Anilins trifft dies in noch erhöhtem Maasse zu. Aber im Uebrigen sind diese Körper recht beständige Verbindungen, die sich z. B. auf dem Wasserbade entweder gar nicht oder nur sehr langsam verandern. Auch durch Eisessig oder Essigsäureanhydrid werden die Anilidoderivate nicht oder nur unbedeutend zersetzt, doch spielt hierbei ihre Făhigkeit, mit Leichtigkeit normale $\mathrm{N}$-Acetate bilden zu können, eine Rolle. (Vergl. unten.)

Ausser diesen beiden Kategorien von Basen sind nur noch die Abkömmlinge des Benzylamins von der Form R.CH.NH. $\mathrm{C}_{7} \mathrm{H}_{7}$ secundüre Amine. Die Haltbarkeit dieser Verbindungen gegen Eisessig ist wesentlich geringer, denn in der Hitze werden sie von diesem Agens durchgehend zersetzt. Dagegen sind sie gegen kochende Laugen ganz oder nahezu bestăndig, übertreffen also in dieser Beziehung die Anilidokörper. Auch auf dem Wasserbade bleiben sie unverändert.

Ihr Verhalten gegen Essigsäureanhydrid kommt nicht in Betracht, da sie sofort in normaler Weise acetylirt werden.

Die Condensationsproducto der Bromide mit allen übrigen Basen gehören - mit Ausnahme der quaternären Pyridin- und Chinolinderivate - zur Klasse der tertiären Amine. Diese Körper sind im Allgemeinen noch bedeutend zersetzlicher als 
die zuletzt besprochenen Verbindungen, doch bestehen Abstufungen zwischen den Abkömmlingen der einzelnen Basen.

Am widerstandsfähigsten dürften, soweit man nach den bisherigen Versuchen urtheilen kann, die Derivate des Ammoniaks, $\left(\mathrm{R}^{\mathrm{C}} \mathrm{CH}_{2}\right)_{3} \mathrm{~N}$, sein.

Ihnen am nächsten kommen die Producte, die sich vom Methylamin ableiten, $\left(\mathrm{R} . \mathrm{CH}_{2}\right)_{2} \cdot \mathrm{N} \cdot \mathrm{CH}_{3}$. Etwa ebenso haltbar sind die entsprechenden Benzylaminabkömmlinge, während auffallender Weise die analog gebauten Aethylaminderivate viel unbeständiger zu sein scheinen. $O b$ dies allgemein zutrifft, kann nicht behauptet werden, da bisher nur die Tribromide des Pseudocumenols und Mesitols mit Aethylamin combinirt worden sind; aber diese beiden Verbindungen gehören jedenfalls, im Gegensatze zu den Methylaminderivaten der gleichen Pseudophenole, zu den allerzersetzlichsten der bisher dargestellten Condensationsproducte.

Im Allgemeinen haben die Abkömmlinge der secundären aliphatischen Amine Anspruch auf diese Bezeichnung, von denen Diamylaminderivate in grösserer Zahl, Diäthylaminproducte nur in wenigen Vertretern untersucht worden sind. Viele von diesen Verbindungen lassen sich nur unter besonderen Vorsichtsmaassregeln, wozu namentlich Vermeidung jeglicher Erwärmung gehört, darstellen, da sonst ausschliesslich oder überwiegend ihre Zersetzungsproducte, in diesem Falle Diphenylmethanderivate, entstehen.

Wie zersetzlich manche dieser Substanzen sind, geht z. B. daraus hervor, dass das Condensationsproduct aus dem p-Dibrom-p-oxypseudocumylbromid und Diamylamin selbst bei gewöhnlicher Temperatur in indifferenten Mitteln rasch Diamylamin abspaltet. Eine Lösung des Körpers in Chloroform beginnt binnen Kurzem sich gelb zu färben, und beim freiwilligen Verdunsten oder auf Zusatz von Petroläther scheidet sich das betrefiende Diphenylmethanderivat aus. Aehnlich verhält sich das entsprechende Mesitolderivat. 
Beim Erhitzen erleiden diese beiden Substanzen, ebenso der Körper

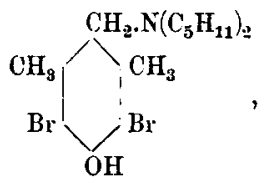

die gleiche Umwandlung so rasch, dass man ihre Schmelzpunkte nur bestimmen kann, indem man das Röhrchen mit der Substanzprobe in ein annähernd auf die Schmelztemperatur erwärmtes Bad bringt.

Auch die Abkömmlinge des Methylanitins sind wenig beständig, doch sind nur vereinzelte Verbindungen dieser Art untersucht worden.

Die in grosser Zahl geprüften Piperidinderivate endlich stehen in Bezug auf ihre Widerstandsfähigkeit zwischen den aus primären - mit Ausnahme von Aethylamin - und secundären aliphatischen Aminen entstehenden Producten.

Mit Pyridin, Chinolin und ähnlichen tertiären Basen treten die Bromide zu quaternären Ammoniumsalzen zusammen, die nicht direct mit den bisher besprochenen Verbindungen vergleichbar sind. Gegen blosses Erhitzen sind sie beständig, werden aber von den verschiedenen chemischen Agentien mit grosser Leichtigkeit zersetzt.

Die bisherigen Versuche lassen somit zur Genüge erkennen, dass die Beständigkeit der hier behandelten Körper in hohem Maasse durch die Natur des basischen Restes beeinflusst wird, wenn auch das diese Beziehungen bestimmende Gesetz noch nicht aufgefunden worden ist.

Um so deutlicher tritt im Grossen und Ganzen der gesetzmässige Zusammenhang hervor, der zwischen der Constitution des phenolischen Restes und dem chemischen Verhalten der Condensationsproducte besteht.

Schon ein flüchtiger Blick auf die Tabellen, namentlich auf Nr. VII und die Uebersicht über die quantitativen Versuche (Nr. VIII), lässt zunächst erkennen, dass die Stellung 
der Seitenkette, in die der basische Rest eingetreten ist, zum Hydroxyl eine massgebende Rolle spielt. Abgesehen von der bemerkenswerthen, unten näher zu erörternden Ausnahmestellung, die die Derivate des Bromids,

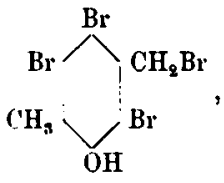

einnelmen, zeichnen sich die Verbindungen der Meta-Reihe durch höchste Beständigkeit aus, denn keines der verschiedenen Agentien vermag sie zu zersetzen. Selbst das Diamylaminderivat,<smiles>CCCCCCCCC(CBr)CBr</smiles>

ist im Gegensatz zu allen ubrigen untersuchten Condensationsproducten dieses Amins vollkommen bestăndig. Ueberraschend ist dieses Verbalten der Meta-Verbindungen keineswegs, denn es entspricht durchaus dem Unterschiede, der zwischen den 0und p-Oxybenzylbromiden mit Pseudophenolcharakter und den m-Bromiden besteht, die sich, von kleinen Eigenthümlichkeiten abgesehen, ähnlich wie gewöhnliche Phenole benehmen.

Nicht ganz so scharf ausgeprägt, aber doch unverkennbar, ist der Unterschied zwischen den Ortho- und Para-Verbindungen. Die Angehörigen der Para-Reihe sind im Allgemeinen bei weitem zersetzlicher. Man vergleiche z. B. das Verhalten der vierfach gebromten Derivate des 0 - und p-Kresols, oder des Monobrom-o- und -p-oxypseudocumylbromids, ferner die $\mathrm{Zu}$ sammenstellung der quantitativen Versuche: kein Ortho-Derivat wird durch kochende Lauge zersetzt, auch wenn es mehrere Methyle im Molektl besitzt. Namentlich gegentber Eisessig und Essigsäureanhydrid macht sich die geringere Beständigkeit der Para-Verbindungen geltend. 
Neben dem eben besprochenen Stellungsunterschiede uben A.t und Zahl der Substituenten im Phenolreste einen nicht minder deutlich erkennbaren Einfluss anf das allgemeine Verhalten der Condensationsproducte aus und diese Verhältnisse bieten $\mathrm{m}$. $\mathrm{E}$. besonderes Interesse.

Sehr gering ist die Wirkung von Bromatomen. Vergleiche zwischen Substanzen, die bei sonst gleicher Constitution sich durch einen verschiedenen Gehalt an Brom unterscheiden, ergeben, dass die bromreicheren Körper zwar durchschnittlich etwas zersetzlicher sind, aber der Unterschied ist verbältnissmässig unbedeutend.

Andere Substituenten, die man als negativ zu bezeichnen pflegt, wie die Aldehyd- und Nitrogruppe, scheinen sogar die Beständigkeit dieser Basen zu erhöhen. Namentlich die Nitrogruppe wirkt anscheinend in diesem Sinne, wie ein Vergleich zwischen den Derivaten der drei Pseudophenole
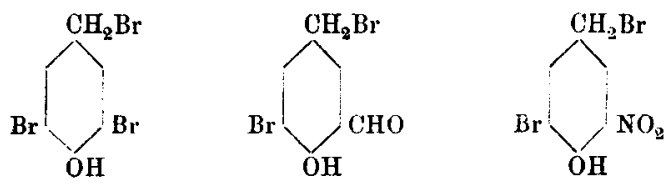

(vergl. Tab. VII) erkennen lässt, denn es ist beispielsweise die Methylaminverbindung dieses Nitrokörpers unter allen untersuchten Para-Derivaten des Methylamins die einzige, die von keinem der angewandten Agentien auch nur im Geringsten zersetzt wird.

Ob wirklich das Nitroradical und Substituenten ähnlichen Charakters regelmässig die Zersetzlichkeit solcher Condensationsproducte vermindern, lässt sich indessen auf Grund der wenigen Versuche, die bisher vorliegen, nicht mit Sicherheit folgern; die Frage soll daher an Verbindungen von bestimmter Constitution, die leider meist recht schwer zugänglich sind, noch weiter geprüft werden.

Eine ganz entgegengesetzte Rolle spielt das Methyl. Es braucht dies hier nicht an einzelnen Beispielen nochmals auf- 
gezeigt zu werden, denn bei der Durcbsicht der Tabellen drängt sich immer von Neuem die Regel auf: je melhr Methyle das Molekïl einer Verbindung enthält, um so zersetelicher ist sie").

Am deutlichsten tritt diese Erscheinung naturgemäss bei den an und für sich weniger beständigen Verbindungen der Para-Reihe zu Tage. Man erkennt bei diesen Körpern auch, dass der Eintritt eines Methyls eine nur măssige Wirkung hervorruft, während durch ein zweites Methyl die Zersetzlichkeit ausserordentlich gesteigert wird. Die dimethylirten Para-Verhindungen besitzen daber unter all diesen Condensationsproducten bei weitem die geringste Bestăıdigkeit.

Sehr klar geht dies auch aus der Tabelle der quantitativen Versuche hervor: die Piperidinderivate der beiden methylfreien Pseudophenole wurden von kochender Natronlauge gar nicht angegriffen; bei den monomethylirten Verbindungen wurden im Laufe einer Stunde rund etwa $10 \mathrm{pC}$., bei den dimethylirten aber 70-90 pC. gespaltet. $\mathrm{Ob}$ auch die Stellung der Methyle von Einfluss ist, bleibt noch eive offene Frage.

Ich habe schon früber ${ }^{6}$ ) kurz darauf hingewiesen, dass auch andere Zersetzungsreactionen durch Methyl in ahnlicher Weise beschleunigt werden und habe zum Theil das betreffende Material

5) Die einzige Ausnahme von dieser Regel bildet das Verhalten der beiden Diamylaminderivate

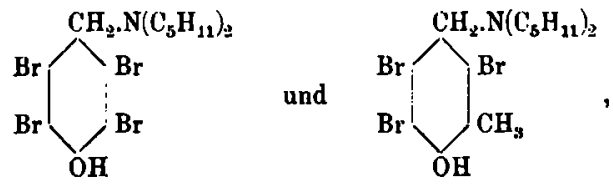

von denen bei den Spaltungsversuchen das erstere in höherem Masse zersetzt wurde. Es war jedoch nicht möglich gewesen, von dieser Substanz ein gut krystallisirtes, reines Präparat zu gowinnen, und so wird denn diese Abweichung vermuthlich auf Rechnung der mangelhaften Reinheit des untersuchten Objectes zu setzen sein.

6) Ber. d. deutsch. chem. Ges. 37, 1473 (1904). 
bereits veröffentlicht ${ }^{7}$ ). Da jedoch eine Reihe von Versuchen, die weitere Beitrăge zur Beleuchtung dieser Frage liefern sollen, noch nicht abgeśchlossen ist, verschiebe ich eine zusammenfassende Besprechung dieser Verbăltnisse, bei der auch die Beobachtungen anderer Forscher heranzuziehen sein werden, auf eine spätere Zeit.

Die bisherigen Versuche haben somit ergeben, dass bei den Coudensationsproducten der Phenolbromide mit organischen Basen alle Stufen von vollkommener Beständigkeit herab bis zu hochgradiger Zersetzlichkeit vorkommen können, ohne dass die Structurformeln dieser Körper derartige Unterschiede ahnen lassen. Auch ein allgemeines Bild von den gesetzmässigen Beziehungen, die zwischen der Constitution dieser Basen und ihrem chemischen Verhalten bestehen, kann man sich auf Grund dieser Versuche machen, doch ist hier noch eine Reihe von Fragen zu lösen und namentlich der quantitative Verlauf, oder besser die Geschwindigkeit der Zersetzungsreactionen genauer zu erforschen.

Ob freilich Bestimmungen, die den an exacte physikalischchemische Untersuchungen zu stellenden Anspruchen genügen, bei diesen Reactionen möglich sein werden, erscheint sehr fraglich, da sie in vielen Fallen nicht hinreichend glatt verlaufen, sondern neben dem Hauptproducte mehr oder weniger gut definirte Nebenproducte entstehen lassen.

Im Allgemeinen wird man bei den verschiedenen Umwandlungen der Condensationsproducte zunächst Abspaltung des Amins und Bildung eines Methylenchinons ${ }^{8}$ ) anzunehmen haben, aus dem dann, je nach den Versuchsbedingungen, Diphenylmethanderivate oder die Acetylverbindungen der zugehörigen Phenolalkohole entstehen können:

7) Ber. d. deutsch. chem. Ges. 32, 3563 (1899).

\$) Vergl. Zincke, diese Annalen $\mathbf{8 2 0}, 145$ (1902! 
<smiles>C=C1C=CC(=O)C=C1</smiles><smiles>C=C1C=NC(=O)C=NC1OCC</smiles><smiles>C=C1C=CC(=O)C=I1</smiles>

I.<smiles>CCC(CCO)CCCCCCO</smiles>

II.<smiles>CCOCCCCCCCCO</smiles>

III.<smiles>CCCCOCCOCC</smiles>

Der stärkste Grund für die Richtigkeit dieser Auffassung liegt darin, dass jede Zersetzung unterbleibt, sobald das Wasserstoffatom im Hydroxyl durch Alkyl oder einen Säurerest ersetzt ist. Stellt man z. B. auf Umwegen die Methyläther oder Acetylverbindungen der in freiem Zustande äusserst zersetzlichen Diamylaminderivate<smiles>CCCCCCC(Br)C(C)Br</smiles>

und<smiles>CCCCCC(Br)(Br)C(C)O</smiles>

dar, so erbält man Substanzen, die völlig beständig gegen alle Operationen und Agentien sind, die nicht etwa eine Abspaltung der am Sauerstoff haftenden Radicale bewirken ${ }^{\mathbf{9}}$ ).

$\left.{ }^{9}\right)$ Diese Versuche sind nicht mit in die Tabellen aufgenommen worden, sind aber in den folgenden Arbeiten beschrieben. 
Fine Ausnahme bilden nur die Pyridin- und Chinolinabkönımlinge, deren Aether und Ester nach dem Schema<smiles>[R1]NC1CCCCC1</smiles>

wie die zugehörigen Phenole, durch Kocben mit Eisessig oder Essigsăureanhydrid glatt nnter Abspaltung des Amins in die Aether und Ester der Bromide verwandelt werden. Diese Reaction der quateruären Verbindungen bat aber mit den Umwandlungen der secundăren und tertiâren Basen nichts zu thun und bietet nichts Auffălliges.

Uebrigens werden die Brombydrate der eben genannten Basen durch siedendes Essigsăureanhydrid in gleicher Weise verăndert; beispielsweise erbalt man aus<smiles>CCN(CC)CCC(C)Br</smiles>

den Kürper<smiles>CCOC(C)C(Br)CBr</smiles>

Weitere derartige Versuche sind in den experimentellen Mittheilungen erwähnt.

Die Wirkung der Substitation am Phenolsauerstoff auf die Beständigkeit der Korper ist nach der oben gegebenen Auffassung des Zersetzungsvorganges ohne weiteres verstandlich, da bei diesen. Derivaten die Bildung eines Methylenchinons verhindert ist.

Bemerkenswerth, weil nicht mit gleicher Sicherheit zu erwarten, erscheint die Thatsache, dass die Anlagerung eines Säurerestes an den Stickstoff eines Condensationsproductes, das den Charakter einer secundären Base hat, die gleiche Wirkung hervorruft. Verbindungen vom Typus

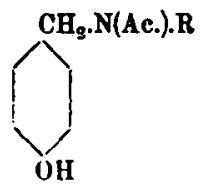


kơnnen beliebig lange mit Laugen, Essigsăure oder Essigsăureanbydrid gekocht werden, ohne Spaltung zu erleiden, obwobl theoretisch ein Zerfall in

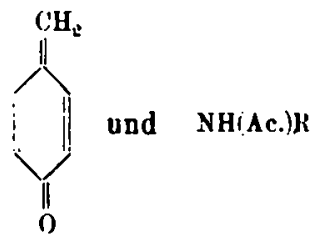

wohl deukhar wăre.

Diese Thatsache ist bei der Beurtheilung der Bestandigkeit mancher Verbindungen gegen saure Agentien zu beracksichtigen. So beruht dic auf den ersten Blick auffallende Widerstandsfahigkeit sänmtlicher Benzylaminderivate vom Schema R. $\mathrm{CH}_{2} \cdot \mathrm{NH}_{7} \mathrm{C}_{7} \mathrm{H}_{7}$ gegen kochendes Essigsảureanhydrid darauf, dass sie fast augenblicklich am Stickstoff acetylirt werden and deshalb hïchstens im ersten Augenblick eine geringfagige Spaltung erleiden.

Auch Eisessig kann sowobl in der Hitze wio in der Kalte in gleicher Weise wirken, und der Verlauf der Spaltungsversuche hägt dann wesentlich davon ab, ob die Geschwindiglscit dieser Acetylirung oder die der Spaltung grosser ist ${ }^{10}$ ).

Sehr merkwturdig ist das Verhalten der Condensationsproducte des dreifach gebromten Mesitols gegen Eisessig. Wăbrend die Abkommlinge sămmtlicher anderen Bromide von siedender Essigsăure, soweit sie tberhaupt von ihr angegriffen werden, entweder in Diphenylmethanderivate oder in die Monoacetate der betreffenden Phenolalkohole verwandelt werden, entstehen aus den Mesitolverbindungen unter den gleichen Bedingungen regelmåssig an Stelle der einfach acetylirten Alkoholo deren Diacetate. Die Versuche sind bei den verschiedensten Verbindungen mehrfach mit gleichem Erfolge wiederholt worden, 80 dass jeder Zweifel ausgeschlossen ist.

17) Vergl. oben Anmerkung 4 . 
Auch bei Zimmertemperatur wirkt 100 procentige Essigsăure auf manche dieser Substanzen überraschend leicht ein; so wird z. B. die Piperidoverbindung<smiles>COC(C)CBr</smiles>

uber Nacht glatt in ihr normales Acetylderivat ubergeführt; enthălt die Essigsăure jedoch etwas Wasser, so findet wie bei analogen Verbindungen keine Einwirkung statt.

Es ist schwer, sich von dem Bildungsmechanismus der Diacetate unter den angegebenen Bedingungen eine Vorstellung zu machen. Die naheliegende Vermuthung, dass zuerst wie in anderen Fallen unter Abspaltung des Amins die Monoacetylverbindung des Phenolalkohols entstele, und diese damn bei weiterem Kochen leichter als analoge Acetate in das Diacetat ubergehe, scheint nicht. zutreffend zu sein, denn die fertige Substanz<smiles>CCOCC(Br)CC(C)O</smiles>

kann Stunden lang mit Eisessig gekocht werden, ohne wesentlich verăndert zu werden. Ebenso verbălt sich ubrigens auch die Stammsubstanz, das Mesitol. Es bliebe dann nur die bedenkliche Annahmie ubrig, dass sich jenes Monoacetat im Augenblicke seines Entstehens gegen kochenden Eisessig anders verhalte als die fertige Verbindung, oder dass sich Essigsäure an das intermediür entstandene Methylenchinon in anderer Weise anlagere als an die abrigen Methylenchinone. Immerhin durfte die letztere Hypothese noch vorzuziehen sein.

Auch noch in anderer Hinsicht ist die Bildung dieser Diacetate auffällig. Wenn, wie oben erwähnt, 100 procentige Essigsänre schon in der Kälte die Piperidoverbindung zu ace- 
tyliren vermag, so sollte man erwarten, dass sich dieser Prozess in der Siedehitze sebr rasch abspielen und damit die Spaltang der Base verhindern warde, denn das fertige Acetat

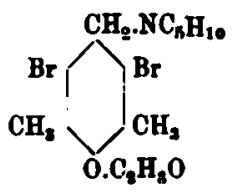

ist gegen nicht verseifend wirkende Agentien ebenso bestăndig wie alle analogen Ester. In Wirklichkeit entsteht aber beim Kochen der Base mit Eisessig glatt das Diacetat des Phenolalkobols und alles Piperidin wird abgespalten. Verstsodlich ist dieser Vorgang nur, wenn man voraussetzt, dass die Spaltung der Base in Methylenchinon und Piperidin anter dem Einfluss der beissen Sarure sehr viel rascher erfolgt als ihre Acetylirung, and dann wieder eine ungewohnliche Art der Anlagerung von Essigsăure stattfindet.

Die sichere Anfklarung dieser abnormen Verhaltnisse bei den Mesitolderivaten, die vermuthlich auch mit der besonderen Constitution dieses Phenols zusammenhăngen, steht somit noch aus.

Auf ahnliche Geschwindigkeitsunterschiede bei concurrirenden Reactionen, wie sie eben erörtert worden sind, mag es zurtackzuftubren sein, dass bei der Einwirkung von Eisesaig und Essigsanresnhydrid auf die verschiedenen Basen bald ausschliesslich oder úberwiegend Mono- und Diacetate von Phenolalkoholen, in anderen Fallen dagegen Diphenylmethanderivate und deren Acetylverbindungen gebildet werden. Auch mogen bei den Versuchen mit Eisessig bin und wieder geringe Concentrationsunterschiede eine Rolle gespielt haben. Nhere Untersuchungen hieruber sind nicht angestellt worden, da diese Fragen for den Zweck der Arbeit nicht wesentlich in Betracht Lamen.

Aus dem, was im Vorstehenden aber die algemeine Art der Einwirkung von Eisessig und Esoigsaureanbydrid an die 
Condensationsproducte mitgetheilt worden ist, ergiebt sich, dass bei diesen Reactionen auf genaue quantitative Bestimmungen kaum zu rechnen ist, da zu viele Factoren ins Spiel kommen, und ferner alle Basen, die leicht in normaler Weise am Stickstoff acetylirt werden können, von vornherein ausscheiden.

Mehr Aussichten auf Erfolg bieten Versuche mit verdünnten Laugen oder reinem Wasser, wenn auch hier neben technischen Unbequemlichkeiten die Schwierigkeit besteht, dass neben den Diphenylmethanderivaten regelmässig auch amorphe Zersetzungsproducte, vermuthlich polymerisirte Methylenchinone, auftreten.

Auch andere indifferente Mittel kämen in Betracht, namentlich Alkohole, von denen Aethylalkohol auf eine Reihe dieser Condensationsproducte bei Siedetemperatur oder im Rohre bei $100^{\circ}$ kräftig spaltend wirkt, während der Einfluss des Methylalkohols geringer zu sein scheint. Einige Beispiele hierfür finden sich in den folgenden Mittheilungen; systematische Versuche sind aber in dieser Richtung noch nicht angestellt worden.

Eine kurze Besprechung erfordern noch die Derivate des Tribrom-m-oxy-p - xylylbromids,

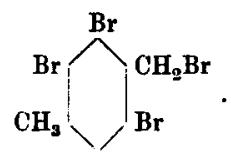

$\mathrm{OH}$

Die Basen, die sich von diesem Bromid ableiten, fallen erstens dadurch auf, dass sie im Gegensatze zu den beiden anderen untersuchten Verbindungen der Meta-Reihe verbältnissmässig wenig beständig sind. (Vergl. Tabelle VII und VIII.) Man könnte geneigt sein, hierin eine Wirkung des paraständigen Methyls zu erblicken, denn dass das Methyl, dessen Einfluss bei all diesen Substanzen so hervorstechend ist, in ParaStellung besonders wirksam sei, wäre nicht auffällig. Indessen hat die, allerdings sehr lückenbafte, Untersuchung der um ein Methyl reicheren Derivate der Bromide 


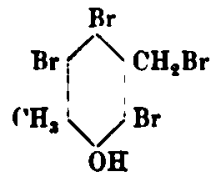<smiles>C[SiH](Br)C(CBr)CBr</smiles><smiles>CC(O)CBr</smiles>

die sămmtlich gleichfalls ein Methyl in derselben Stellung zu der betreffenden Seitenkette enthalten, nichts Aehnliches orgeben.

Der Ausnahmefall bleibt also vorlăufig unerklărlich.

Zweitens verdienen diese Verbindungen desbalb Interesse, weil sie durch kochende Laugen unter Abspaltung von Amin wie die Korper der Ortho- und Para-Reihe in Diphenylmethanderivate verwandelt werden sollen. Eine intermediare Bildung eines Methylenchinons muss aber bei diesen Metaderivaten als ausgeschlossen betrachtet werden.

Da bei den Abkð̋mmlingen anderer Metabromide diese Reaction nicht durchgefuhrt werden konnte ${ }^{11}$ ), habe ich diese Versuche, die von Herrn A. Schrenk angestellt worden sind, gomeinsam mit Herrn Dr. 0 . Schröter am Piperidinderivat

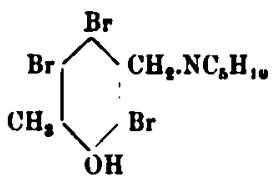

'J) Die Angabe des Herrn R. Zaubitzer, Dissertation Seite 28, dasa das Diamylaminderivat von der Formel<smiles>OC(Br)[C@H](Br)[C@H](Br)[Mg]Br</smiles>

durch kochende Lauge in das Octobrom - mm - dioxydiphenylmethan<smiles>Br[Mg]Br</smiles><smiles>CC(Br)(Br)Br</smiles>

übergetührt werde, hat sich als gänzlich falsch erwiesen. 
mit besonderer Sorgfalt nachgeprüft. Dic Thatsache der leichten Spaltbarkeit und alle sonstigen Schrenk'schen Angaben konnten im Wesentlichen bestängt werden, doch stellte es sich heraus, dass das durch Kochen mit Laugen erbaltene Spaltungsproduct zum allergrössten Theile aus amorphen, unlöslichen Substanzen bestand. Die Menge des Alkalilöslichen war bei unseren Versuchen so gering, dass wir auf eine Identificirung verzichten mussten. Auch Herr Schrenk hatte bei den einzelnen Spaltungen so wenig von diesem Körper gewonnen, dass er schliesslich aus sämmtlichen Versuchen nur so viel des reinen Körpers darstellen konnte, als für eine Analyse erforderlich war.

Es ist daher anzunehmen, dass sich bei der Zersetzung jener Basen zwar in ganz untergeordneter Menge ein Diphenylmethanderivat bildet, dass jedoch dic Reaction wesentlich anders wie bei den Verbindungen der Ortho- und Para-Reihe verläuft, die zunächst in ein Methylenchinon übergehen können.

In den Tabellen ist das erwähnte Diphenylmethanderivat mit einem Fragezeichen versehen worden, da seine Existenz zwar sehr wahrscheinlich ist, aber doch noch der Bestätigung bedarf.

Zum Schluss sei noch kurz zweier Reihen von Versuchen gedacht, die noch weiterer Durcharbeitung bedürfen.

Die eine Gruppe beschäftigt sich mit den halogenfreien Oxybenzylpiperidinen von der Form

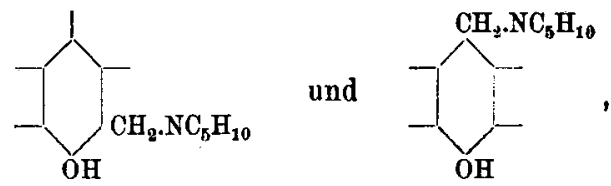

die nach den Vorschriften des D. R. P. Nr. 89979 durch gleichzeitige Einwirkung von Formaldehyd und Piperidin auf bestimmte Phenole gewonnen werden können. Mau hatte gehofft, an diesen Substanzen, die als weitere Substituenten lediglich Alkylgruppen enthalten, den Einfluss von Art, Zahl und Stellung der Alkyle auf die Spaltbarkeit dieser Basen noch ge- 
nauer feststellen zu können, als an den bis dahin ausschliesslich untersuchten halogenhaltigen Körpern. Diese Erwartung hat sich indessen bis jetzt nicht erfullt, denn ein Theil dieser Substanzen ist ziemlich löslich in Wasser, vor allem aber uberraschend leicht mit Wasserdumpfen fluchtig, so dass sich die Trennung der in der Regel entstehenden Gemische von unzersetzter Base und abgespaltenem Piperidin nicht in allen Fallen mit gentigender Schärfe hat durchfuhren lassen.

Da zudem die Versuche bis jetzt nur von einem Beobachter, Herrn A. Dombrowski, angestellt und noch nicht von anderer Seite nachgepruft worden sind, soll vorläufig von ihrer Wiedergabe abgesehen werden.

Bei der anderen Serie von Versuchen, die gleichfalls von Herrn A. Dombrowski ausgeführt worden sind, handelte es sich darum, zu ermitteln, ob die Einführung von Methyl in die basische Hälfte des Molekuls der hier behandelten Condensasionsproducte ebenso wirke, wie der Eintritt dieses Radicals in den Phenolrest. $\mathrm{Zu}$ diesem Zweck condensirte man das Tribromderivat des Pseudocumenols mit Anilin, den drei Toluidinen, den sechs Xylidinen, sowie Pseudocumidin und prufte die entstandenen Basen auf ihre Spaltbarkeit.

Soweit diese Versuche bis jetzt reichen, lassen sie im Allgemeinen eine Zunahme der Zersetzlichkeit mit steigender Zahl der Methyle erkennen, doch scheinen starke Abweichungen von dieser Regel vorzukommen. Namentlich erwecken die bisher gewonnenen Ergebnisse den Eindruck, als ob bei diesen Korpern neben der Zahl auch die Stellung der Methyle eine bedeutende Rolle spiele, und die Verhältnisse wesentlich verwickelter seien, als bei den fruber untersuchten Verbindungen.

Diese Versuche bedurfen daher einer besonders sorgfăltigen Prufung; namentlich sind hier exacte Messungen in grosser Zahl erforderlich, wenn man zu völlig sicheren Ergebnissen gelangen will.

Ein Theil des experimentellen Materials, das die Grundlage-der in -dieser Arbeit gegebenen Ausfuhrungen bildet, ist 
in den Dissertationen der Herren A. Dombrowski, C. Kipke, A. Schrenk und O. Schröter ${ }^{12}$ ) niedergelegt, doch haben die späteren Nachprüfungen zum Theil zu abweichenden Ergebnissen gefübrt. Die folgenden Mittheilungen sind zum grossen Theil auf Grund dieser späteren Untersuchungen bearbeitet worden und enthalten ausserdem noch weiteres Material. Die Beschreibung der noch nicht bekannten Verbindungen und die analytischen Belege sind vollständig gegeben; Einzelbeiten über die Darstellung mancher Körper, sowie über die Durchführung einer Reihe von Versuchen können aus den Dissertationen ersehen werdeu.

\section{Ueber Condensationsproducte}

von organischen Basen mit Phenolen und Pseudophenolen der Kresolreihe;

$$
\text { von } K \text {. Auwers und } O \text {. Schröter. }
$$

Die Derivate der einfachsten bromirten Pseudophenole sind zuerst von Herrn R. Zaubitzer ${ }^{1}$ ) untersucht worden. Indessen haben sich bei einer Nachprüfung seine Beobachtungen und Angaben als so unzuverlässig erwiesen, dass wir die ganze Árbeit in allen Einzelheiten wiederholen mussten. Was sich von den Zaubitzer'schen Untersuchungen als zutreffend erwiesen hat, ist in diese Mittheilung übernommen worden.

Bei der Darstellung der Ausgangsmaterialien und der einzelnen Condensationsproducte sind wir von Herrn Dr. C. Roever wesentlich unterstützt worden.

12) Greifswald $1903-1905$.

1) Inaug.-Dissert. Greifswald 1904. 


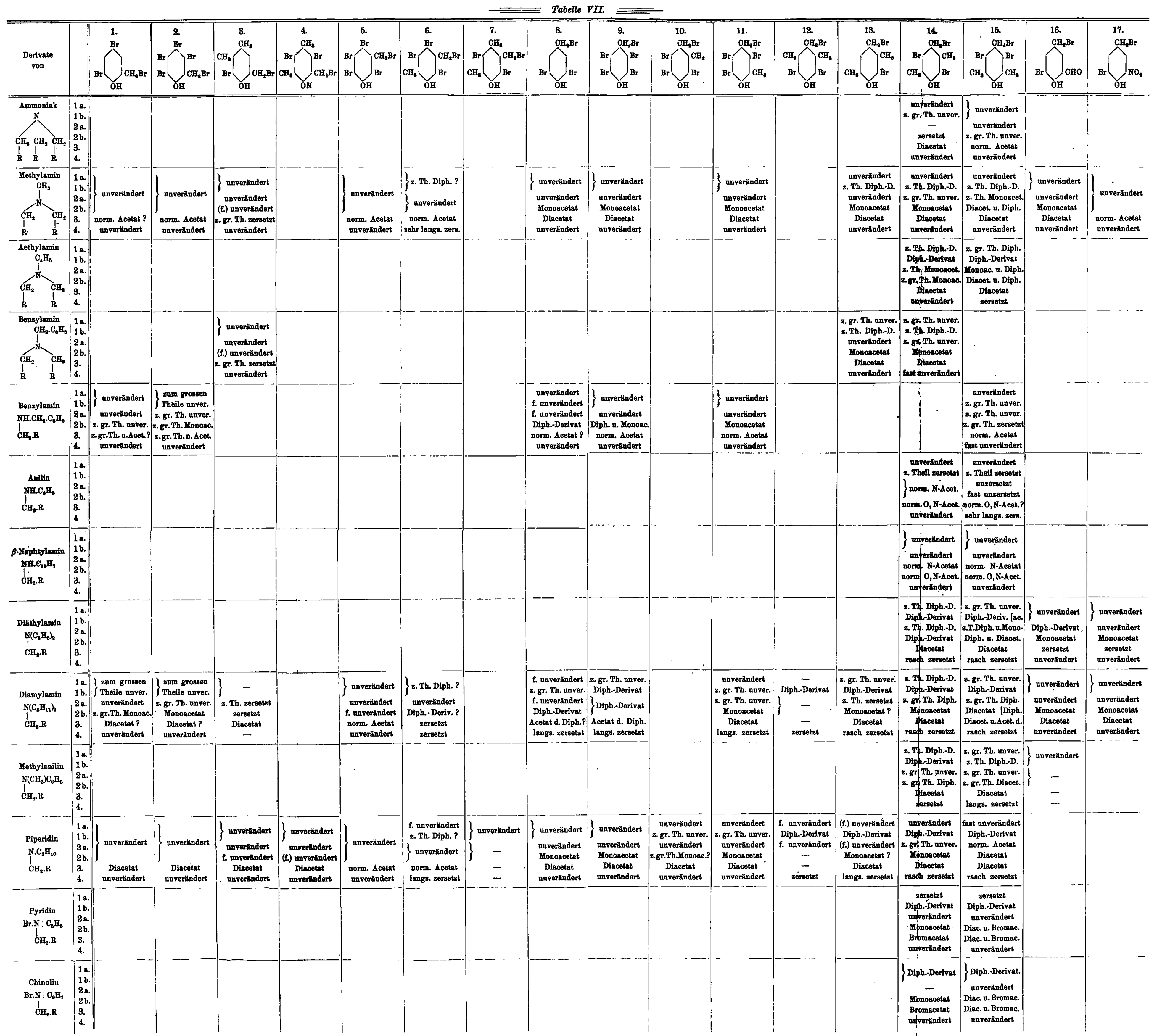

